\title{
Emergence of Adaptive Computation by Single Neurons in the Developing Cortex
}

\author{
Rebecca A. Mease, ${ }^{1,2}$ Michael Famulare, ${ }^{3}$ Julijana Gjorgjieva, ${ }^{4}$ William J. Moody, ${ }^{5}$ and Adrienne L. Fairhall ${ }^{6}$ \\ ${ }^{1}$ Neurobiology and Behavior Graduate Program, University of Washington, Seattle, Washington 98195, ${ }^{2}$ Institute of Neuroscience, Technische Universität \\ München, D-80333 Munich, Germany, ${ }^{3}$ Department of Physics, University of Washington, Seattle, Washington $98195,{ }^{4}$ Center for Brain Science, Harvard \\ University, Cambridge, Massachusetts 02138, and ${ }^{5}$ Department of Biology and ${ }^{\circ}$ Department of Physiology and Biophysics, University of Washington, \\ Seattle, Washington 98915
}

Adaptation is a fundamental computational motif in neural processing. To maintain stable perception in the face of rapidly shifting input, neural systems must extract relevant information from background fluctuations under many different contexts. Many neural systems are able to adjust their input- output properties such that an input's ability to trigger a response depends on the size of that input relative to its local statistical context. This "gain-scaling" strategy has been shown to be an efficient coding strategy. We report here that this property emerges during early development as an intrinsic property of single neurons in mouse sensorimotor cortex, coinciding with the disappearance of spontaneous waves of network activity, and can be modulated by changing the balance of spike-generating currents. Simultaneously, developing neurons move toward a common intrinsic operating point and a stable ratio of spike-generating currents. This developmental trajectory occurs in the absence of sensory input or spontaneous network activity. Through a combination of electrophysiology and modeling, we demonstrate that developing cortical neurons develop the ability to perform nearly perfect gain scaling by virtue of the maturing spike-generating currents alone. We use reduced single neuron models to identify the conditions for this property to hold.

\section{Introduction}

Many neural systems adjust their input-output properties in response to changes in the statistical properties of the incoming stimulus. Through adaptation, the nervous system continually recalibrates its sensitivity under new contexts to best represent the range of inputs it receives (Attneave, 1954; Barlow, 1961; Laughlin, 1981; Atick, 2011). One form of such adaptive computation is gain scaling, whereby a neural system's mapping between inputs and outputs adjusts to dynamically span the varying range of incoming stimuli (Brenner et al., 2000; Fairhall et al., 2001; Kim and Rieke, 2001; Ringach and Malone, 2007). In this form of adaptive coding, the nonlinear function relating input to output has the property that the gain with respect to the input scales with the SD of the input. While circuit mechanisms are often thought to be responsible for gain scaling (Wark et al., 2007;

Received July 5, 2012; revised May 13, 2013; accepted May 16, 2013.

Author contributions: R.A.M., W.J.M., and A.L.F. designed research; R.A.M., M.F., and A.L.F. performed research; R.A.M., M.F., and J.G. analyzed data; R.A.M., M.F., J.G., W.J.M., and A.L.F. wrote the paper.

This work was funded by a National Institutes of Health Institutional Grant for Neurobiology T32 GM07108 -35 (R.A.M.), McKnight Scholar Award in the Neurosciences (A.L.F., M.F.), National Science Foundation (NSF) Grant EF0928251 (M.F., J.G., A.L.F.), and NSF IOB0718344 (W.J.M.). We thank A. McCabe, J. Lischalk, C. Easton, and J. Conhaim for culture preparation; A. Groh for electrophysiology assistance; B. Lundstrom and H. Barnett for critical discussions; L. Matkins for computing advice; and B. Sakmann for allowing R.A.M. to complete a portion of the experiments as a guest in his laboratory.

Correspondence should be addressed to either of the following: Rebecca A. Mease, Institute of Neuroscience, Technische Universität München, D-80802 Munich, Germany, E-mail: rebecca.mease@|rz.tum.de; or Adrienne L. Fairhall, Department of Physiology and Biophysics, University of Washington, Seattle, WA 98195, E-mail: fairhall@uw.edu.

DOI:10.1523/JNEUROSCI.3263-12.2013

Copyright $\odot 2013$ the authors $\quad 0270-6474 / 13 / 3312154-17 \$ 15.00 / 0$
Carandini and Heeger, 2012), we show here that single cortical neurons adjust their dynamic range to changes in the typical size of input fluctuations. We investigate the specific biophysical properties that underlie this adaptive computation in single neurons.

While gain scaling is widely observed in neural systems (Wark et al., 2007), little is known about how it develops in neurons. During maturation of neural circuits in the cortex, synaptic connections change in number and in sign (Sanes et al., 2006). Simultaneous with synaptic development, the biophysical properties of cortical neurons change as the densities of ion channels change (Picken-Bahrey and Moody, 2003a; Moody and Bosma, 2005); we show that this developmental progression also alters how neurons encode synaptic inputs into output spikes. Here, we investigated the impact of developmental changes in intrinsic properties on information processing by cortical neurons.

We measured the gain-scaling properties of individual neurons in the developing mouse sensorimotor cortex over two age ranges: embryonic day 18 to postnatal day 1 (E18-P1, "immature," and P6-P8, "mature”). These developmental stages bridge a major transition in cortical excitability-while immature neurons engage in slow waves of network-wide spontaneous activity (Picken-Bahrey and Moody, 2003a; Corlew et al., 2004; McCabe et al., 2006), mature neurons display nascent adult firing properties (McCormick and Prince, 1987; Connors and Gutnick, 1990) and are no longer spontaneously active (Corlew et al., 2004). A hallmark of this transition is the ongoing expression of voltagegated sodium, $I_{\mathrm{Na}}$, and potassium, $I_{\mathrm{K}}$, channels (McCormick and 
Prince, 1987; Picken-Bahrey and Moody, 2003b) that comprise the basic spike-generating mechanism (Hodgkin and Huxley, 1952). The corresponding currents appear early in the development of cortical neurons: $I_{\mathrm{K}}$ is present in the stem cell population of the ventricular zone before the first neurons exit the cell cycle, and $I_{\mathrm{Na}}$ can be detected even before differentiating neurons migrate into the cortical plate (Picken-Bahrey and Moody, 2003b).

We have shown previously that $I_{\mathrm{Na}}$ increases in density much faster than $I_{\mathrm{K}}$ during early postnatal development (PickenBahrey and Moody, 2003a). Here, we examine how these changes in the maturing spike-generation mechanism impact the adaptive computational properties of cortical neurons by using whole-cell current-clamp and voltage-clamp recordings in vitro in acute and organotypic slices. We find that cortical neurons acquire the ability to perform nearly perfect gain scaling solely through developmental changes in the expression of spikegenerating sodium and potassium currents, without significant evidence or need for slow adaptation currents or conductance changes. Using pharmacology and the analysis of reduced neuronal models, we show how gain control may be implemented solely by the simplest spike-generating dynamics.

\section{Materials and Methods Electrophysiology}

We recorded intracellularly from single neurons in layers II-VI of mouse sensorimotor cortex at two time points: from E18 to P1 (organotypic culture, $n=15$ ), and from P6 to P8 (organotypic culture, $n=41$; acute slices: $n=19$ ).

\section{Tissue preparation}

Organotypic cultures. Organotypic cultures were prepared from embryonic (E17) Swiss-Webster and BALB/C mouse cortex from either sex as previously described by (McCabe et al., 2006). According to our previous work in organotypic cultures, this preparation preserves the normal development of single neuron intrinsic properties and has the advantage of allowing block of early spontaneous network activity via application of tetrodotoxin (TTX) from E18 to P3 (for review, see McCabe et al., 2006). All procedures were in accordance with National Institutes of Health guidelines, and were approved by the Institutional Animal Care and Use Committee of the University of Washington.

Acute slices. While the majority of recordings were done in cultured slices, we also performed a series of experiments $(n=19)$ in acute slices. Black-6 mouse pups (P6-P8) were killed by exposure to $\mathrm{CO}_{2}$ and decapitated. Brains were rapidly removed from the skulls and cerebellar regions dissected before gluing the remaining tissue to the vibratome plate (MicroM HM 650V; Thermo Scientific). Coronal slices (300 $\mu \mathrm{M})$ were made in ice-cold artificial CSF (ACSF; identical to recording solution described below, but with $50 \mu \mathrm{M} \mathrm{CaCl}{ }_{2}$ ). Before recording, slices were incubated in $34^{\circ} \mathrm{C} \mathrm{ACSF}$ with added synaptic blockers (see below) for at least $30 \mathrm{~min}$. All animal handling followed animal welfare guidelines for the Technical University of Munich.

\section{Patch-clamp recording conditions}

For experiments in Swiss-Webster neurons, patch pipettes were pulled to a resistance of 3.5-8 M $\Omega$ from $50 \mu \mathrm{l}$ hematocrit glass capillary tubes using a two-stage puller (Narishige), and filled with potassium internal solution, which contained the following (in mM): $113 \mathrm{KMeSO}_{4}$ (ICN Biomedicals), 28 $\mathrm{KCl}, 10$ HEPES, 2 ATP-Mg, $3 \mathrm{Na}_{2}$-ATP, and 0.2 Na-GTP, pH to 7.25. During recording, tissue was maintained in room-temperature ACSF bubbled with carbogen gas $\left(95 \% \mathrm{O}_{2}-5 \% \mathrm{CO}_{2}\right)$ containing the following (in $\left.\mathrm{mM}\right): 140$ $\mathrm{NaCl}, 3 \mathrm{KCl}, 2 \mathrm{MgCl}_{2}, 2 \mathrm{CaCl}_{2}, 1.25 \mathrm{NaHPO}_{4}, 26.5 \mathrm{NaHCO}_{3}$ and 20 D-glucose. All salts were obtained from Sigma. Recordings were made using a List EPC-7 (Heka Elektronik) amplifier. Currents were filtered at $1.5 \mathrm{kHz}$ with an 8-pole Bessel characteristic, recorded and analyzed using pCLAMP8 and pCLAMP9 software (Molecular Devices).

For experiments in the BALB/C and Black- 6 neurons, patch pipettes for whole-cell recordings were filled with the following (in $\mathrm{mm}$ ): 105
K-gluconate, $30 \mathrm{KCl}, 10$ HEPES, 4 ATP-Mg, 10 phosphocreatine- $\mathrm{Na}_{2}$, and $0.3 \mathrm{NaGTP}, \mathrm{pH}$ to 7.3. The extracellular ACSF solution contained the following (in mM): $125 \mathrm{NaCl}, 2.5 \mathrm{KCl}, 1 \mathrm{MgCl}_{2}, 2 \mathrm{CaCl}_{2}, 1.25 \mathrm{NaHPO}_{4}$, $25 \mathrm{NaHCO}_{3}$, and 25 glucose. Recordings were made with an Axoclamp 2B amplifier (Molecular Devices), filtered at $3 \mathrm{kHz}$, and acquired using custom-written software (Igor; WaveMetrics).

During all experiments, neurons were synaptically isolated by extracellular application of $20 \mu \mathrm{M} \mathrm{AP5}, 25 \mu \mathrm{M}$ CNQX, and $10 \mu \mathrm{M}$ picrotoxin or $50 \mu \mathrm{M}$ bicuculline. For some neurons, $1 \mathrm{~mm} 4$-aminopyridine (4-AP) was applied to reduce a transient potassium current. For other neurons, $5 \mathrm{~nm}$ TTX was applied to partially block the transient sodium current responsible for generating action potentials. All blockers were from Tocris Bioscience.

\section{Voltage-clamp protocol}

Input resistance $r_{i n}$ was calculated from the linear fit to steady-state currents at voltages from -80 to $-40 \mathrm{mV}$ ( $\pm 10 \mathrm{mV}$ steps from a holding potential of $-70 \mathrm{mV}$ ), sampled at $20 \mathrm{kHz}$. The magnitudes of the maximal sodium and potassium currents, denoted $I_{\mathrm{Na}}$ and $I_{\mathrm{K}}$, were estimated from current responses to voltage steps ranging from -80 to $+40 \mathrm{mV}$ from a holding potential of $-70 \mathrm{mV}$ (see Fig. $3 D$ ), after leak subtraction (Picken-Bahrey and Moody, 2003a). Maximal $I_{\mathrm{Na}}$ was measured at its peak value; maximal $I_{\mathrm{K}}$ was measured at $+40 \mathrm{mV}, 70 \mathrm{~ms}$ after the beginning of the step, after sodium currents had inactivated. Series resistance was typically $8-10 \mathrm{M} \Omega$ and compensated by at least $60 \%$.

Due to space clamp issues resulting from currents in the dendrites and axon, it is unlikely that we achieved perfect control over the applied voltage. However, we do not believe that a systematic, stage-dependent change in spatial control of voltage biases our results, for several reasons. First, at least proximal neurites are present throughout the stages of our experiments and the growth of more distal processes at later stages would not be expected to have large effects on spatial control (Picken-Bahrey and Moody, 2003a). Second, $I-V$ relations for the voltage-gated $\mathrm{Na}$ and $\mathrm{K}$ currents rise smoothly at all stages, indicating that major escapes in membrane potential are not occurring, and, most relevant to our experiments, are not affecting $\mathrm{Na}$ and $\mathrm{K}$ currents differentially. In cases where voltage escapes were apparent, data were not accepted. Finally, the developmental increase in $\mathrm{Na}$ current density observed in this and previous work was closely correlated with predicted changes in basic firing properties and action potential waveform, indicating that the observed changes are not simply artifacts of poor and changing spatial control (Picken-Bahrey and Moody, 2003a).

\section{Current-clamp noise stimulation}

For spike-triggered characterization (see below), broadband Gaussian noise current, $i(t)$, with mean, $\mu$, and $\mathrm{SD}, \sigma$, was injected to elicit long $(1000-2000 \mathrm{~s})$ spike trains, acquired in $100 \mathrm{~s}$ trials. All input current traces were realizations of the Ornstein-Uhlenbeck process (Risken, 1996), expressed as follows:

$$
i(t)=\mu+\sigma \xi(t),
$$

where $\xi(t)$ has unit variance and correlation time ( $\tau_{c}=1 \mathrm{~ms}$ always $)$. During noise trials, no holding current was applied; neurons with resting membrane potentials positive to $-60 \mathrm{mV}$ were not analyzed further. MATLAB 7 was used to create stimulus waveforms (digitized at 1 or 2 $\mathrm{kHz}$ ) and for custom data analysis routines. For single $\sigma$ experiments, $\sigma$ was increased until the neuron fired repetitively at $\sim 5-10 \mathrm{~Hz}$ while holding $\sigma=2 \mu$ to maintain the relative shape of the input current distribution between neurons with large differences in passive input resistance. In multiple $\sigma$ experiments used to measure gain scaling, we started with $\sigma=2 \mu$ and then increased $\sigma$ while holding $\mu$ constant. For most neurons, gain scaling was tested at $\sigma=1.0$ and $1.3, n=25$; for a subset of neurons, a wider range of SDs was tested $(\sigma=1.0,1.3,2.0$, and 2.6), $n=$ 7. To further probe the gain-scaling ability during partial sodium channel block, some neurons in these experiments were tested at $\sigma=1.0,1.3$, and $1.5, n=12$. For small correlation times, when $\tau_{c}$ is much smaller than the membrane time constant $\tau_{\mathrm{v}}$, the input can be thought of as white noise that has been discretized in samples of duration $\tau_{\mathrm{c}}$ (Risken, 1996). Here, $\tau_{\mathrm{c}} / \tau_{\mathrm{v}}$ is typically approximately $1 / 20$; in our models, a larger $\tau_{\mathrm{c}}$ does not 
qualitatively change any reported results (data not shown). The scale of voltage fluctuations driven by the current input can be expressed as follows:

$$
\sigma_{v}=r \sigma \sqrt{\frac{\tau_{\mathrm{c}}}{\tau_{\mathrm{v}}}}
$$

where $r$ is the input resistance. In the recordings shown, $4 \mathrm{mV} \widetilde{<} \sigma_{V} \widetilde{<} 20$ $\mathrm{mV}$.

The mean current has a significant effect on the response properties of the cell. For all ages studied, without a positive mean current, resting potentials were approximately $v_{\text {rest }}=-70 \mathrm{mV}$ with thresholds near -30 $\mathrm{mV}$, making it difficult to drive spiking without unphysiological ranges of $\sigma$. The DC input $\mu$ moved the effective resting potential from approximately $v_{\text {rest }}=-70 \mathrm{mV}$ with thresholds near $-30 \mathrm{mV}$ into the range -55 to $-40 \mathrm{mV}$ without substantially changing the threshold. This mean input replaces the slow background depolarizations (hundreds of milliseconds and longer; slow relative to the $20 \mathrm{~ms}$ timescale of the relevant stimulus; see Fig. $1 B$ ) that would be present due to spontaneous network activity if synapses were not blocked (Picken-Bahrey and Moody, 2003a; McCabe et al., 2006). For a summary of the data from mature P7 cells, see Table 2.

Exponential integrate-and-fire (EIF) models were fit to a population of mature cells (P7, $n=6)$ to quantitatively characterize the neuronal dynamics in a framework amenable to theory. Fits were determined manually from the statistics of the voltage distribution and the spike train in response to a known input current. All cells included were statistically stationary for at least $100 \mathrm{~s}$ for each input condition (exhibited stable mean voltage and mean firing rate); nonstationary data (observed to vary on timescales of 50-100 s) were excluded from the fits. Goodness of fit to the spike train was determined by the coincidence factor (Kistler et al., 1997). Goodness of fit of the linear-nonlinear (LN) models resulting from the best-fit EIF model was determined by $\bar{D}_{M}$, the symmetrized Kullback-Leibler (KL) divergence, averaged over $\sigma$, between the spiketriggered, normalized filtered stimulus distributions, $p[\hat{s} \mid \mathrm{sp}]$ for the recorded data and the best-fit EIF model.

\section{Representing intrinsic computation with LN models}

We characterized the intrinsic computation of individual in vitro or model neurons as a one-dimensional LN cascade model (Hunter and Korenberg, 1986) calculated via reverse correlation (de Boer and Kuyper, 1968) of output spike times to the input Gaussian current stimulus with $\mathrm{SD} \sigma$ (Figure 1).

An LN model for a single neuron produces an estimate of the instantaneous firing rate in response to the input current. The LN model consists of two parts: a feature that acts on the input to produce a linearly filtered stimulus $s(t)$, the amplitude of the feature present in the input, and a nonlinear input-output relation that maps the filtered stimulus $s(t)$ to the instantaneous firing rate. We take the feature to be the spiketriggered average (STA), denoted $h$. This filter $h$ has length $T$ and is normalized to have unit gain (Rieke et al., 1996). Thus, the relevant component of the stimulus is as follows:

$$
s(t)=\int_{0}^{T} d t^{\prime} h\left(t^{\prime}\right)\left(i\left(t-t^{\prime}\right)-\mu\right) \equiv\left(h^{\star} i\right)(t)
$$

With this choice of normalization, the variance of the filtered stimulus is linearly proportional to the variance of the unfiltered input current: $\left\langle s(t)^{2}\right\rangle \propto \sigma^{2}$.

The neuron's selectivity for the identified feature is expressed by the nonlinear input-output relation, $R[s] . R[s]$ is related to the probability that the input is associated with spikes, $p[s \mid \mathrm{sp}]$, by Bayes' law:

$$
\begin{aligned}
R[s] & \equiv \frac{P[\mathrm{sp} \mid s]}{d t} \\
& =\bar{R} \frac{p[s \mid \mathrm{sp}]}{p[s]},
\end{aligned}
$$

where $\bar{R}$ is the mean firing rate for fixed input mean and SD $\sigma, \mathrm{d} t$ is the sampling time step, and the prior distribution $p[s]$ is always Gaussian with mean zero and variance $\sigma^{2}$. The spike-triggered stimulus distribution $p[s \mid \mathrm{sp}]$ is found by computing the histogram of the filtered stimulus values, $s$, given the event that a spike occurs, which we denote sp. These quantities are generally a function of the statistics of the input; here we vary the SD. This is indicated by a subscript: $\bar{R}_{\sigma}$ and $R_{\sigma}(s)$.

\section{Gain scaling}

The input-output relation generally depends on the input statistics, $\mu$ and $\sigma$; therefore, across input conditions, an individual neuron is described by a family of LN models. For systems with perfect gain scaling, at a fixed mean $\mu$, gain scaling occurs if the input-output relations for different $\sigma$ (when expressed in terms of normalized inputs $\hat{s} \equiv s / \sigma$ ) factor into a fixed $\sigma$-independent normalized response function, $\hat{R}[s / \sigma] \equiv \hat{R}[\hat{s}]$, and a $\sigma$-dependent multiplicative gain:

$$
R_{\sigma}[s]=\bar{R}_{\sigma} \hat{R}[\hat{s}]
$$

Note that the rescaling property is a change in "input gain," whereby the effective dynamic range of the inputs is scaled; a change in the multiplicative prefactor (here, the mean firing rate $\bar{R}$ ) is known as "output gain" (Carandini and Heeger, 2012).

This property can be equivalently expressed using the spikeconditional filtered stimulus distribution, Equation 4, as the stimulus distribution $p(s)$ scales with $\sigma$ by construction. Gain scaling then requires that the spike-triggered filtered stimulus distribution is independent of $\sigma$ when expressed in terms of the scaled stimulus $\hat{s}$, so that for inputs with two different SDs $\sigma_{1}$ and $\sigma_{2}$,

$$
p_{\sigma_{1}}[\hat{s} \mid \mathrm{sp}]=P_{\sigma_{2}}[\hat{s} \mid \mathrm{sp}]
$$

where $p_{\sigma}$ denotes the distribution of filtered stimuli given that the input is drawn from a Gaussian distribution with $\mathrm{SD} \sigma$, by the Jacobian rescaling of distributions under a change of variable, $p_{\sigma}[\hat{s} \mid \mathrm{sp}]=\sigma p_{\sigma}[s \mid \mathrm{sp}]$.

\section{Quantifying gain scaling}

We take advantage of Equation 4 to quantify the degree of gain scaling using the spike-triggered stimulus distributions directly. We use the symmetrized KL divergence (Lin, 1991) of the spike-triggered stimulus distributions in terms of the scaled stimulus, where $\hat{s}$ is scaled by the SD denoted by the subscript of its function as follows:

$$
D_{\sigma}=\frac{1}{2} \int d \hat{s}\left(p_{\sigma_{1}}[\hat{s} \mid \mathrm{sp}] \log _{2}\left(\frac{p_{\sigma 1}[\hat{s} \mid \mathrm{sp}]}{p_{\sigma 2}[\hat{s} \mid \mathrm{sp}]}\right)+p_{\sigma_{2}}[\hat{s} \mid \mathrm{sp}] \log _{2}\left(\frac{p_{\sigma_{2} 2}[\hat{s} \mid \mathrm{sp}]}{p_{\sigma 1}[\hat{s} \mid \mathrm{sp}]}\right)\right) .
$$

For $D_{\sigma \rightarrow 0}$ bits, gain scaling is perfect. This approach avoids parameter fitting and makes no assumptions about the shape of the distributions. Histograms were typically discretized at 0.1 normalized stimulus units. Due to finite sample size, empty bins in the histograms $p_{\sigma}[s \mid s p]$ are possible toward the tails; a small value (MATLAB variable eps) was added to such bins.

We also use Equation 6 to compare the input/output relations from different neurons. We define $D_{\mathrm{N}}$ as the symmetrized divergence between $p_{\sigma}[\hat{s} \mid s p]$ sampled for two different neurons; small $D_{\mathrm{N}}$ indicates that the two neurons have the same input-output function shape. To quantify the difference between the mature and immature neurons with respect to the variability of the threshold functions within each maturity level, we compared the distributions of pairwise $D_{\mathrm{N}}$. As Figure $3 C$ demonstrates, the distributions of $D_{\mathrm{N}}$ for immature and mature neurons are not Gaussian, so we use a twosided Kolmogorov-Smirnov test to compute $p$ values. Unless otherwise noted, all other significance tests were unpaired two-sided $t$ tests; $\langle\cdot\rangle$ indicates a mean value; error bars are the SEM.

To ensure that empty bins in the sampled $p[s \mid$ sp] distributions did not largely impact our results from Equation 6, we also calculated the mean Jensen-Shannon divergence, $\bar{D}_{J S}$, between distributions by calculating the symmetrized KL divergence between each $p_{\sigma}[\hat{s} \mid \mathrm{sp}]$ distribution and the mean distribution, $m=\frac{1}{2}\left(p_{\sigma_{1}}[\hat{s} \mid \mathrm{sp}]+P_{\sigma_{2}}[\hat{s} \mid \mathrm{sp}]\right)$, and then taking the average of the two resulting values: 


$$
\bar{D}_{I S}=\frac{1}{2} \int d \hat{s}\left\langle p_{\sigma}[\hat{s} \mid \mathrm{sp}] \log _{2}\left(\frac{p_{\sigma}[\hat{s} \mid \mathrm{sp}]}{m[\hat{s}]}\right)+m[\hat{s}] \log _{2}\left(\frac{m[\hat{s}]}{p_{\sigma}[\hat{s} \mid \mathrm{sp}]}\right)\right\rangle_{\sigma}
$$

$\bar{D}_{J S}$ scaled linearly with $D_{\sigma}$; thus, our particular choice of metric did not affect the trends on which we based our conclusions.

The $D_{\sigma}$ values we calculated for most mature neurons were quite small ( $\sim 0.1-0.3$ bits). During low gain conditions, especially during the drug (4-AP and TTX) experiments, there were a relatively small number of recorded spikes (i.e., $<1000$ spikes). For small numbers of spikes, finite sampling and choice of bin size used to compute distributions might create artifacts in $D_{\sigma}$ calculations: one expects artificially high values of $D_{\sigma}$ due to undersampling of an underlying smooth distribution. To examine the expected finite data size effects, we computed individual experimental $p[\hat{s} \mid \mathrm{sp}]$ distributions by dividing the data randomly into two halves, $p^{(1)}[\hat{s} \mid \mathrm{sp}]$ and $p^{(2)}[\hat{s} \mid \mathrm{sp}]$, and calculated $D_{\sigma}$ between these distributions; this value should be very close to zero. For the range of bin sizes we used in our calculations, the resulting average sampling error was between 0.06 and 0.08 bits; this sampling error applies for all reported $D_{\sigma}$, as calculations were performed with matched spike counts. Thus, the majority of small $D_{\sigma}$ values indicating precise gain scaling were close to the $D_{\sigma}$ values, which arise from sampling alone. These margins of error were in agreement with those estimated via bootstrap resampling (2000 repetitions), which ranged from 0.008 to 0.072 bits (mean 0.032 bits, 18 neurons, $30 \%$ gain change).

\section{Single neuron models}

Using single compartment models, we examined the impact of different maximal sodium and potassium conductances on the properties of the computation as characterized by LN models.

\section{Biophysical model}

In close parallel with the experiments, we studied a Hodgkin-Huxley style model (Hodgkin and Huxley, 1952) consisting of a passive leak current, $i_{\mathrm{L}}$, and mammalian voltage-gated transient sodium $i_{\mathrm{Na}}$, and a delayed-rectifier potassium, $i_{\mathrm{K}}$, currents with corresponding maximal conductances, $G_{\mathrm{L}}, G_{\mathrm{Na}}$, and $G_{\mathrm{K}}$, and reversal potentials $E_{\mathrm{L}}=-70 \mathrm{mV}$, $E_{\mathrm{Na}}=50 \mathrm{mV}$, and $E_{\mathrm{K}}=-77 \mathrm{mV}$ :

$$
\begin{aligned}
C \dot{v} & =-i_{\mathrm{L}}-i_{\mathrm{Na}}-i_{\mathrm{K}}+i(t), \\
& =G_{\mathrm{L}}\left(E_{\mathrm{L}}-v\right)+g_{\mathrm{Na}}(t)\left(E_{\mathrm{Na}}-v\right)+g_{\mathrm{K}}(t)\left(E_{\mathrm{K}}-v\right)+i(t),
\end{aligned}
$$

where $C=1 \mu \mathrm{F} / \mathrm{cm}^{2}$ is the specific membrane capacitance and $i(t)$ is the input current. The active conductances are given in terms of the gating variables $m, h$, and $n$ :

$$
\begin{gathered}
g_{\mathrm{Na}}=G_{\mathrm{Na}} m^{3} h, \\
g_{\mathrm{K}}=G_{\mathrm{K}} n .
\end{gathered}
$$

The kinetics of the gating variables are as follows:

$$
\begin{aligned}
& \tau_{m}(v) \dot{m}=m_{\infty}(v)-m, \\
& \text { with } m_{\infty}(v)=\frac{\alpha_{m}(v)}{\alpha_{m}(v)+\beta_{m}(v)} \text { and } \tau_{m}(v)=\frac{1}{\alpha_{m}(v)+\beta_{m}(v)} ; \\
& \tau_{h}(v) \dot{h}=h_{\infty}(v)-h, \\
& \text { with } h_{\infty}(v)=\frac{1}{1+\exp \left(\frac{v-V_{h}}{K_{h}}\right)} \text { and } \tau_{h}(v)=\frac{1}{\alpha_{h}(v)+\beta_{h}(v)} ;
\end{aligned}
$$

Table 1. Kinetic parameters of the biophysical model

\begin{tabular}{llcll}
\hline Variable & Equation & $A_{x}\left[10^{-3}\right]$ & $V_{x}[\mathrm{mV}]$ & $K_{x}[\mathrm{mV}]$ \\
\hline$m$ & $\alpha_{m}$ & 182 & -35 & 9 \\
& $\beta_{m}$ & 124 & -35 & 9 \\
$h$ & $\alpha_{h}$ & 24 & -50 & 5 \\
& $\beta_{h}$ & 9.1 & -75 & 5 \\
& $h_{\infty}$ & - & -65 & 6.2 \\
$n$ & $\alpha_{n}$ & 20 & 20 & 9 \\
& $\beta_{n}$ & 2 & 20 & 9 \\
\hline
\end{tabular}

$$
\text { with } n_{\infty}(v)=\frac{\alpha_{n}(v)}{\alpha_{n}(v)+\beta_{n}(v)} \text { and } \tau_{n}(v)=\frac{1}{\alpha_{n}(v)+\beta_{n}(v)} .
$$

where the rate coefficients, $\alpha_{\mathrm{x}}(v)$ and $\beta_{\mathrm{x}}(v)$, are of the form:

$$
\alpha_{x}(v)=\frac{A_{\alpha_{x}}\left(v-V_{\alpha_{x}}\right)}{1-\exp \left(-\frac{v-V_{\alpha_{x}}}{K_{x}}\right)} \text { and } \beta_{x}(v)=\frac{-A_{\beta_{x}}\left(v-V_{\beta_{x}}\right)}{1-\exp \left(\frac{v-V_{\beta_{x}}}{K_{x}}\right)} .
$$

The kinetic parameters are shown in Table 1, from Mainen et al. (1995). For compactness, $m_{\infty}(v)$ and $n_{\infty}(v)$ can be re-expressed as follows:

$$
x_{\infty}(v)=\frac{1}{1+e^{-\left(\frac{v-V_{x}-\Delta V_{x}}{K_{x}}\right)}}
$$

with $V_{x} \equiv V_{\alpha \mathrm{x}}\left(=V_{\beta \mathrm{x}}\right)$ and $\Delta V_{x}=K_{x} \ln \left(\frac{A_{\beta_{x}}}{A_{\alpha_{x}}}\right)$.

Simulations were performed in the NEURON simulation environment (Hines and Carnevale, 1997). Maximal conductances $G_{\mathrm{Na}}$ and $G_{\mathrm{K}}$ ranged over $100-2000 \mathrm{pS} / \mu \mathrm{m}^{2}$. To match the range of input resistances seen in vitro (0.2-1.5 G $\Omega$; see Picken-Bahrey and Moody (2003a) for discussion of the high input resistance in developing cortical neurons), the leak conductance was set to $G_{\mathrm{L}}=0.25 \mathrm{pS} / \mu \mathrm{m}^{2}$ such that the membrane time constant at the resting potential was $25 \mathrm{~ms}$. Of 400 possible conductance combinations 217 produced model neurons that were not spontaneously active and fired in response to noisy current. Figure $6 \mathrm{~A}$ details the model's excitability as function of $G_{\mathrm{Na}}$ and $G_{\mathrm{K}} ; 20,000$ spikes were used for all calculations, with an integration step $\mathrm{d} t=0.1 \mathrm{~ms}$. Ten simulations with different $G_{\mathrm{Na}} / G_{\mathrm{K}}$ were repeated for $\mathrm{d} t=0.01 \mathrm{~ms}$, with minor changes in spike timing and no changes in LN models. As discussed in the main paper, gain scaling of the LN models improves with increasing $G_{\mathrm{Na}} / G_{\mathrm{K}}$, as shown in Figure $6 B$.

\section{Exponential integrate and fire model}

We performed theoretical analysis and fits to data with the exponential integrate and fire (EIF) model, a single variable model that has been shown to fit well to data from cortical neurons (Badel et al., 2008a,b). The EIF model replaces Hodgkin-Huxley style dynamics with nonlinear dynamics given by an exponential dependence on $V$ that drives spiking and an instantaneous after-spike reset that terminates the action potential:

$$
\tau_{V} \dot{v}=v_{o}-v+f(v)-\left(v_{s}-v_{r}\right) \tau_{V} R(t)+r \sigma \xi(t)
$$

with

$$
\begin{aligned}
R(t)=\sum_{\left\{t_{s}\right\}} \delta\left(t-t_{s}\right) \text { where }\left\{t_{s} \in t: v(t)=\right. & \left.v_{s}\right\}, \\
f(v)=\left(v_{t h}-v_{o}\right)\left(e^{\frac{v-v_{t h}}{\Delta}}\right. & \left.-\left(1+\frac{v-v_{o}}{\Delta}\right) e^{\frac{v-v_{t h}}{\Delta}}\right) \\
& \times\left(1-\left(1+\frac{v_{t h}-v_{o}}{\Delta}\right) e^{\frac{v-v_{t h}}{\Delta}}\right)^{-1}
\end{aligned}
$$

The parameter $v_{o}$ is the effective resting potential for mean input $\langle i(t)\rangle=\mu$ in the sense used in Equation 16, $\tau_{\mathrm{v}}$ is the effective membrane time constant near rest as in Equation 17, $v_{s}$ is the peak voltage of the spike, $v_{r}$ is the reset voltage immediately after a spike, and $r$ is the input 
resistance. The instantaneous firing rate $R(t)$ drives the voltage reset from $v_{s}$ to $v_{r}$ at every spike time, when $v$ reaches $v_{s}$.

The function $f(v)$ is the exponential voltage-activated current, and $\Delta$ sets the activation scale over which the spike-driving excitable current turns on. For fixed $\mu$ (which has been absorbed into the definition of $v_{o}$ ), there is an unstable fixed point at $v_{t h}$ that acts as the intrinsic dynamical threshold for pulse-like inputs (Gerstner and Kistler, 2002; Hong et al., 2007; Izhikevich, 2007; Famulare and Fairhall, 2010, 2011). This form of $f(v)$ (Fourcaud-Trocmé et al. (2003)) has been chosen so that each parameter retains its intuitive meaning regardless of the parameter values. Parameterized as above, it is assumed that the neuron exhibits a stable resting state and threshold, i.e., is excitable. Furthermore, as $f(v)$ has the properties $f\left(v_{o}\right)=0, f\left(v_{t h}\right)=v_{t h}-v_{o}$, and $d f / d v\left(v_{o}\right)=0$ for all values of the activation parameter $\Delta$, this parameterization allows us to vary $\Delta$ without also changing the directly measurable, model-independent parameters, $v_{o}, v_{t h}$, and $\tau_{\mathrm{v}}$.

Five of the parameters determine units and the finite cutoff for the spike height (to which the model is quite insensitive; Touboul, 2009). The two important dependencies are the dimensionless ratios describing the spike generating currents: $\Delta / v_{t h}-v_{o}$ and $v_{r}-v_{o} / v_{t h}-v_{o}$. The input fluctuation strength is captured similarly in terms of $\sigma_{v} / v_{t h}-v_{o}$.

\section{Spike threshold}

For neurons in the low $\mathrm{Na}^{+}$conductance regime and for the corresponding EIF parameters, spikes are broad and it is necessary to choose a criterion to define the spike time. We chose to define $v_{\text {spike }}$ statistically as the voltage beyond which at least $95 \%$ of trajectories continue to spikes; spike times are defined as the instants that $v_{\text {spike }}$ is crossed from below. As a strong hyperpolarizing input fluctuation can abort a spike, $v_{\text {spike }} \geq v_{t h}$. Although $v_{\text {spike }}$ is then weakly $\sigma$ dependent, the gain-scaling property of the sampled input-output functions does not depend on the definition (see Fig. 9; it also holds robustly for $\sigma$-independent $v_{\text {spike }}$ ). The threshold definition affects which voltage fluctuations are classified as spikes, and so slightly affects the measured overall firing rate and the filter shape.

\section{Biophysical interpretation of the EIF model parameters}

We reparametrize the conductance-based model, Equation 8, to aid in interpreting the EIF model parameters. First, we explicitly separate the linearized response around the effective resting potential from the full nonlinear dynamics. For input currents with nonzero mean, $\langle i(t)\rangle=\mu$, the effective resting potential is as follows:

$$
v_{o}=\frac{G_{L} E_{L}+g_{N a}\left(v_{o}\right) E_{N a}+g_{K}\left(v_{o}\right) E_{K}+\mu}{G_{L}+g_{N a}\left(v_{o}\right)+g_{K}\left(v_{o}\right)}
$$

where $g_{\mathrm{Na}}\left(v_{o}\right)$ and $g_{\mathrm{K}}\left(v_{o}\right)$ are the steady-state conductances at the effective resting potential. For small $G_{\mathrm{L}}, v_{o}$ only depends on the maximal conductance ratio, $G_{\mathrm{Na}} / G_{\mathrm{K}}$, and not the absolute conductance values. The effective input resistance at $v_{o}$ is $r=\left(G_{\mathrm{L}}+g_{\mathrm{Na}}\left(v_{o}\right)+g_{\mathrm{K}}\left(v_{o}\right)\right)^{-1}$, and the corresponding effective membrane time constant is as follows:

$$
\tau_{V}=\frac{C}{G_{L}+g N a\left(v_{o}\right)+g_{K}\left(v_{o}\right)}
$$

In terms of $v_{o}, \tau_{\mathrm{v}}$, and $\sigma$, Equation 8 becomes:

$$
\tau_{V} \dot{v}=v_{o}-v+r \delta g_{\mathrm{Na}}(t)\left(E_{\mathrm{Na}}-v\right)+r \delta g_{K}(t)\left(E_{\mathrm{K}}-v\right)+r \sigma \xi(t),
$$

where the conductance differences are defined as $\delta g_{\mathrm{X}}(\mathrm{t})=g_{\mathrm{X}}(t)-g_{\mathrm{X}}\left(v_{o}\right)$. Averaging over the channel dynamics conditioned on the instantaneous value of the voltage gives a one-dimensional model in the voltage:

$$
\tau_{V} \dot{v}=v_{o}-v+r\left\langle\delta g_{\mathrm{Na}} \mid v\right\rangle\left(E_{\mathrm{Na}}-v\right)+r\left\langle\delta g_{K} \mid v\right\rangle\left(E_{\mathrm{K}}-v\right)+r \sigma \xi(t),
$$

Numerical (Badel et al., 2008a,b) and limited analytical (Abbott and Kepler, 1990; Kepler et al., 1992; Jolivet et al., 2004) methods are available for performing the averaging explicitly. This reduction is most accurate when the conductance states are tightly correlated with the instantaneous value of the voltage, as occurs when the timescales of the conductance dynamics are much faster than the timescale of membrane dynamics.
This is approximately true for the biophysical model for the parameters simulated, except during the afterpolarization, when the details of the spike waveform are not important to the questions we studied (below threshold: $\left.\tau_{\mathrm{v}}=40 \mathrm{~ms}, \tau_{m} \sim 0.3 \mathrm{~ms}, \tau_{n} \sim 7 \mathrm{~ms}, \tau_{h} \sim 10 \mathrm{~ms}\right)$.

In the EIF model, the function $f(v)$ that approximates the nonlinear currents that are active below threshold and during spike generation, Equation 15, is characterized by the voltage threshold, $v_{t h}$, and the activation parameter, $\Delta$. The threshold voltage in the EIF model characterizes the separation between the subthreshold and spiking regimes and is approximately the unstable fixed point of the averaged dynamics:

$$
f\left(v_{t h}\right)=v_{t h}-v_{o} \approx r\left\langle\delta g_{\mathrm{Na}} \mid v_{t h}\right\rangle\left(E_{\mathrm{Na}}-v_{t h}\right)+r\left\langle\delta g_{K} \mid v_{t h}\right\rangle\left(E_{\mathrm{K}}-v_{t h}\right) ;
$$

only approximate equality can be expected because any simple form chosen for $f(v)$ is unlikely to perfectly match the true averaged kinetics. The activation parameter, $\Delta$, primarily captures the kinetics of sodium activation (Fourcaud-Trocmé et al., 2003), although the best-fit value is influenced by all active nonlinearities preceding a spike. The reset to $v_{r}$ when the voltage exceeds a spike height, $v_{s}$, replaces biophysical dynamics for the afterpolarization. When the mean interspike interval is large compared with the effective membrane time constant, $\tau_{\mathrm{v}}$, the EIF model is insensitive to $v_{r}$ when $v_{r}$ is such that

$$
v_{o} \sim v_{r} \lesssim v_{t h}-\Delta \text { and } \Delta \lesssim v_{t h}-v_{o}
$$

To understand how gain scaling in the reduced model relates to that in the conductance-based model, we examine how $v_{t h}$ and $v_{o}$ vary with changes in the maximal conductance ratio, $G_{\mathrm{Na}} / G_{\mathrm{K}}$. From Equation 19, the derivative of the threshold voltage with respect to changes in the maximal conductance ratio is as follows:

$$
\frac{\partial v_{t h}}{\partial\left(\frac{G_{\mathrm{Na}}}{G_{\mathrm{K}}}\right)}=\frac{\frac{G_{\mathrm{K}}}{G_{\mathrm{Na}}} r \delta g_{\mathrm{Na}}\left|v_{t h}\right\rangle\left(E_{\mathrm{Na}}-v_{t h}\right)}{1-\frac{\partial}{\partial v}\left[r\left\langle\delta g_{\mathrm{Na}} \mid v\right\rangle\left(E_{\mathrm{Na}}-v\right)+r\left\langle\delta g_{K} \mid v\right\rangle\left(E_{K}-v\right)\right]_{v=v_{t h}}},<0,
$$

where the inequality follows because the numerator is positive-definite and the denominator is negative-definite since the voltage threshold is dynamically unstable (Izhikevich, 2007). Thus, as the maximal conductance ratio is increased, the voltage threshold decreases, as is well known since the sodium channel is responsible for excitability. The derivative of Equation 16 with respect to the conductance ratio will have the opposite sign because the resting potential is dynamically stable: $\frac{\partial v_{o}}{\partial\left(\frac{G_{\mathrm{Na}}}{G_{\mathrm{K}}}\right)}>0$. Thus, an increase in the maximal conductance ratio decreases the distance between rest and threshold:

$$
\frac{\partial\left(v_{t h}-v_{o}\right)}{\partial\left(\frac{G_{\mathrm{Na}}}{G_{\mathrm{K}}}\right)}<0
$$

This correspondence can be seen by comparing the behavior of the full biophysical model (see Fig. 7) to that of the EIF model (see Fig. 10).

For fixed spike-generating kinetics, represented by fixed $\Delta$, increasing the conductance ratio in the biophysical model (and increasing the maximal current ratio in the recorded neurons) is equivalent to increasing $\Delta /\left(v_{t h}-v_{o}\right)$ in the EIF model. Qualitatively, $\Delta /\left(v_{t h}-v_{o}\right)$ captures in a single parameter the interaction of the kinetics of spike initiation $(\Delta)$ with developing expression of ion channels $\left(v_{t h}-v_{o}\right)$.

\section{Fitting the EIF model to data}

EIF models were fit to a population of mature cells (P7, $n=6)$ probed by four different input SDs. An EIF-specific algorithmic model fitting approach (Badel et al., 2008a,b) with electrode correction (Brette et al., 2007) was attempted but failed due to the insufficient sampling rate (2 $\mathrm{kHz}$ ), and so fits were performed manually. Initial parameter estimates 
Table 2. Population summary of relevant parameters for mature cortical cells

\begin{tabular}{lr}
\hline Parameter & Mean value \pm STD \\
\hline resting potential $(\mu=0)$ & $-68.5 \pm 2.5 \mathrm{mV}$ \\
$\mu$ & $39.9 \pm 17.4 \mathrm{pA}$ \\
$v_{o}(\mu \neq 0)$ & $-48.2 \pm 5.4 \mathrm{mV}$ \\
$v_{t h}$ & $-25.3 \pm 1.1 \mathrm{mV}$ \\
$\tau_{\mathrm{v}}$ & $18.0 \pm 4.2 \mathrm{~ms}$ \\
$\Delta$ & $9.3 \pm 2.3 \mathrm{mV}$ \\
$v_{r}$ & $-55.4 \pm 7.1 \mathrm{mV}$ \\
$v_{s}$ & $37.0 \pm 3.5 \mathrm{mV}$ \\
$r$ & $150 \pm 90 \mathrm{M} \Omega$ \\
\multicolumn{1}{c}{$\Delta$} & $0.37 \pm 0.05$ \\
$v_{\text {th }}-v_{o}$ & \\
$\frac{v_{r}-v_{o}}{v_{\text {th }}-v_{o}}$ & $-0.1 \pm 0.2$ \\
\hline P7; $\mathrm{no}$ DC input $(\mu=0): n=28 ;$ with DC input: $n=6$. &
\end{tabular}

were derived from the STA $\left(\tau_{v}\right)$ and steady-state voltage distribution $\left(v_{o}\right.$, $v_{t h}, v_{r}, v_{s}, r$, and $\left.\Delta\right)$. From the initial estimates, parameters were varied to optimize the model fit to the STA, fit to the subthreshold steady-state voltage distribution, and coincidence factor. All cells included were statistically stationary (as determined by the mean firing rate and mean voltage, averaged in $500 \mathrm{~ms}$ blocks) for at least $100 \mathrm{~s}$ for each input condition; nonstationary data (observed to vary on timescales of 50-100 s) were excluded from the fits.

The spike height voltage, $v_{s}$, was taken to be the maximum voltage observed, although all results are insensitive to this choice provided $v_{s}-$ $v_{t h} \gg \Delta$ (Fourcaud-Trocmé et al., 2003; Touboul, 2009). The initial guess for the effective membrane time constant, $\tau_{v}$, was matched to the timescale of the STA. Initial guesses for the kinetic parameters were set to $\Delta=0.3$ and $v_{r}-v_{o}=0$. Given the above choices, initial values for the effective resting potential, $v_{o}$, threshold, $v_{t h}$, and resistance, $r$, were determined by optimizing the fit of the steady-state voltage distribution below threshold:

$$
p_{\sigma}[v]=\frac{2 \bar{R}_{\sigma} \tau_{V}}{\sigma_{v}^{2}} e^{\frac{\left(v-v_{0}\right)^{2}-2 F(v)}{\sigma_{v}^{2}}} \int_{\max \left(v, v_{r}\right)}^{v_{s}} d v^{\prime} e^{\frac{\left(v^{\prime}-v_{o}\right)^{2}-2 F\left(v^{\prime}\right)}{\sigma_{v}^{2}}},
$$

where $F(v)=\int_{-\infty}^{v} \mathrm{~d} u f(u)$ and the mean rate, $\bar{R}_{\sigma}$, is found from the normalization condition, $[\mathrm{d} v p[v]=1$ (Fourcaud-Trocmé et al., 2003; Paninski et al., 2003).

From the initial guess, systematic variation of parameters was used to optimize the mean coincidence factor across all input conditions. The coincidence factor, applied to a single input condition, is as follows:

$$
\Gamma=\frac{N_{\text {coinc }}-\left\langle N_{\text {coinc }}\right\rangle}{\frac{1}{2}\left(N_{\text {data }}+N_{\text {model }}\right)} \mathcal{N}^{-1}
$$

where $N_{\text {coinc }}$ is the number of spikes that coincide within a tolerance $\pm \gamma$, $\left\langle N_{\text {coinc }}\right\rangle=2 \bar{R}_{\sigma} \gamma N_{\text {data }}$ is the expected number of coincidences for a Poisson spike train with the same rate as the data, and $\mathcal{N}^{-1}=1-2 \bar{R} \gamma$ is a normalization factor (Kistler et al., 1997). The coincidence factor is zero for random Poisson coincidence and is one for spike trains that agree exactly. The tolerance was chosen to be one mean spike width, $\gamma=7 \pm 3$ $\mathrm{ms}$ for the population (slow spikes are consistent with the small conductances seen at this age (Picken-Bahrey and Moody, 2003a).

Best-fit parameters for the population are summarized in Table 2. Across the population, the mean coincidence factor for an input condition was $\langle\Gamma\rangle=0.59 \pm 0.07$ (the statistical error for a condition is \pm 0.02 ). For each cell, the EIF model identified was used to generate LN models corresponding to the recorded data. Simulation results are shown in Figure 8. Input-output function similarity between model and data was characterized by the mean Jensen-Shannon divergence between model and data, $\bar{D}_{\mathrm{M}}$, defined as in Equation 7. Population statistic is $\left\langle\bar{D}_{\mathrm{M}}\right\rangle=0.36 \pm 0.19$ bits across all input conditions; estimated floor is 0.1 bits, based on random resampling as above.

\section{Results}

To characterize the computation of a neuron, we constructed LN models by stimulating the cell body with Gaussian noise current scaled by a range of SDs and recording the resulting spike times (Bryant and Segundo, 1976; Agüera y Arcas et al., 2003). In the LN model (Fig. 1), the computation is represented as linear feature selection and nonlinear encoding: from its input, the neuron selects the relevant signal component by linear filtering - the filtered stimulus, $s(t)$ - and a nonlinear input-output relation determines the instantaneous firing rate from the filtered stimulus, $R[s(t)]$ (see Materials and Methods; Hunter and Korenberg (1986)). We identified the neuron's preferred feature as its STA, (Fig. 1B; Eq. 3), the mean time-varying current input preceding a spike (Bryant and Segundo, 1976; Rieke et al., 1996). Across different stimulus conditions, any changes in a neuron's intrinsic transformation of current inputs to output spike times can in principle appear as changes in the STA or in the shape of the input-output relation.

The input currents were realizations of a Gaussian noise process with mean $\mu, \mathrm{SD} \sigma$, and $1 \mathrm{~ms}$ correlation time, $\tau_{\mathrm{c}}$, Equation 1. We focus on adaptation to the SD and hold the mean input fixed. The filter is defined to have a gain of unity so that the amplitude of the filtered stimulus $s(t)$ is proportional to the amplitude of the input current $I(t)$, and all changes in gain appear as changes in the input-output relation, $R[s]$. To study gain scaling, we define the scaled, normalized input-output relation, $\hat{R}_{\sigma}[\hat{s}] \equiv R[\hat{s}] / \bar{R}$, where $\hat{s}=s / \sigma$ and $\bar{R}_{\sigma}$ is the mean firing rate at a given $\sigma$.

To test the gain-scaling properties of single neurons, we compared $\hat{R}_{\sigma}[\hat{s}]$ obtained using noisy current stimuli with different SDs. If perfect gain scaling occurs between two stimulus conditions $\sigma_{1}$ and $\sigma_{2}$, the input-output relations adapt to the range given by the stimulus SD such that the stimulus is encoded in units relative to $\sigma: \hat{R}_{\sigma_{1}[\hat{s}]}=\hat{R}_{\sigma_{2}[\hat{s}]}$ (Brenner et al., 2000; Fairhall et al., 2001; Famulare and Fairhall, 2011). In contrast, if the neuron uses the same input-output relation independent of $\sigma$, the scaled relations will differ significantly. In general, one expects that neural systems will realize coding properties that lie between these extremes (Gaudry and Reinagel, 2007). We quantified differences in $\hat{R}_{\sigma}[\hat{s}]$ for different $\sigma$ using an information measure, $D_{\sigma}$ (Fig. $1 C$; Eq. 6). A neuron that shows perfect scaling for $\sigma_{1}$ and $\sigma_{2}$ will have $D_{\sigma}$ close to zero (see Materials and Methods).

\section{Gain scaling in single neurons}

We begin by demonstrating gain scaling in a single cortical neuron at P7 (an example from the "mature" group) stimulated with noise of different SDs (Fig. 2). The STAs had a consistent shape across the range of $\sigma$ (Fig. $2 A$ ): the STA is dominated by a peak of depolarizing current immediately before the spike, typically preceded by a shallow hyperpolarizing trough. This general shape is typical for STAs calculated for vertebrate central neurons (Mainen et al., 1995; Svirskis et al., 2003; Powers et al., 2005; Slee et al., 2005). Typically, the corresponding input-output relations, $R_{\sigma}[s]$, were exponential functions of stimulus amplitude for small $s$ but saturated for large $s$ (Fig. $2 B$ ). The midpoint of each $R_{\sigma}[s]$ increased with $\sigma$. However, when plotted with respect to the stimulus scaled by $\mathrm{SD}, \hat{s}=s / \sigma$, the input-output relations $\hat{R}_{\sigma}[\hat{s}]$ were nearly identical (Fig. $2 C$ ). Through gain scaling (small $D_{\sigma}$ ), the neuron maintains the same form of response nonlinearity independent of the SD of the stimulus distribution. While the data shown in Figure 2 is from a neuron from organotypic culture, we found similar gain-scaling results in neurons from acute slices of the same age (data not shown). 


\section{Convergence to a common}

input-output relation and ratio of spike-generating currents

We next compared the shapes of the STAs and input-output relations between the mature (P6-P8) and immature (E18-P1) neuron groups. Each neuron was driven with a single noise stimulus, with $\sigma$ chosen such that neurons from both stages fired at similar rates $(5-10 \mathrm{~Hz})$. These stimulus amplitudes varied over an order of magnitude in absolute units, reflecting the diverse excitability of the population, particularly the decrease in passive input resistance which occurs over this developmental period (Picken-Bahrey and Moody, 2003a). Typically, immature STAs had a broader temporal integration window than mature STAs (Fig. $3 A$ ). The scaled input-output relations of all mature neurons, $\hat{R}_{\sigma}[\hat{s}]$, were remarkably consistent (Fig. $3 B$ ): not only did the response properties of mature neurons scale for stimuli of different $\sigma$, these neurons shared a common population function $\hat{R}_{\sigma}[\hat{s}]$. In contrast, $\hat{R}_{\sigma}[\hat{s}]$ of immature neurons had a smaller stimulus domain, saturated at lower stimulus values, and were more variable between neurons. We quantified the difference between two neurons' input-output relations for the same stimulus $\mathrm{SD}$ as $D_{\mathrm{N}}$, analogous to $D_{\sigma}$ (see Materials and Methods and Eq. 6). The mean immature $D_{\mathrm{N}}$ was significantly greater than that of mature neurons (Fig. 3C), reflecting the variability seen between individual immature neurons' inputoutput relations. Furthermore, mean error in gain scaling $\left(\left\langle D_{\sigma}\right\rangle\right.$; see Materials and Methods) was smaller for mature neurons (red, $\left\langle D_{\sigma}\right\rangle=0.26 \pm 0.04$ bits, $n=11$ ) than for immature neurons (blue, $\left\langle D_{\sigma}\right\rangle=0.56 \pm 0.10$ bits, $n=6, p=0.0019)$. Thus, the ability of cortical neurons to scale response gain to match input statistics emerges during the first week of postnatal development, along with the convergence to a common population input-output relation.

We next characterized the intrinsic biophysical parameters that might underlie these properties. During embryonic and early postnatal development, the relative densities of spike-generating channels change. Na channels increase in density much faster than delayed K channels (Picken-Bahrey and Moody, 2003b) and thus support regenerative membrane depolarization underlying action potential generation. Using a voltage-clamp protocol (see Materials and Methods and Fig. 3D), we measured the maximal spike-generating currents, denoted $I_{\mathrm{Na}}$ and $I_{\mathrm{K}}$, throughout early cortical development for neurons ranging from E14 to P11. During development, the ratio of $I_{\mathrm{Na}}$ to $I_{\mathrm{K}}$ initially increases with age, and then converges to a constant value after P0 (Fig. $3 E$ ). These results suggest the increase in $\mathrm{Na}$ to $\mathrm{K}$ current ratios might un-
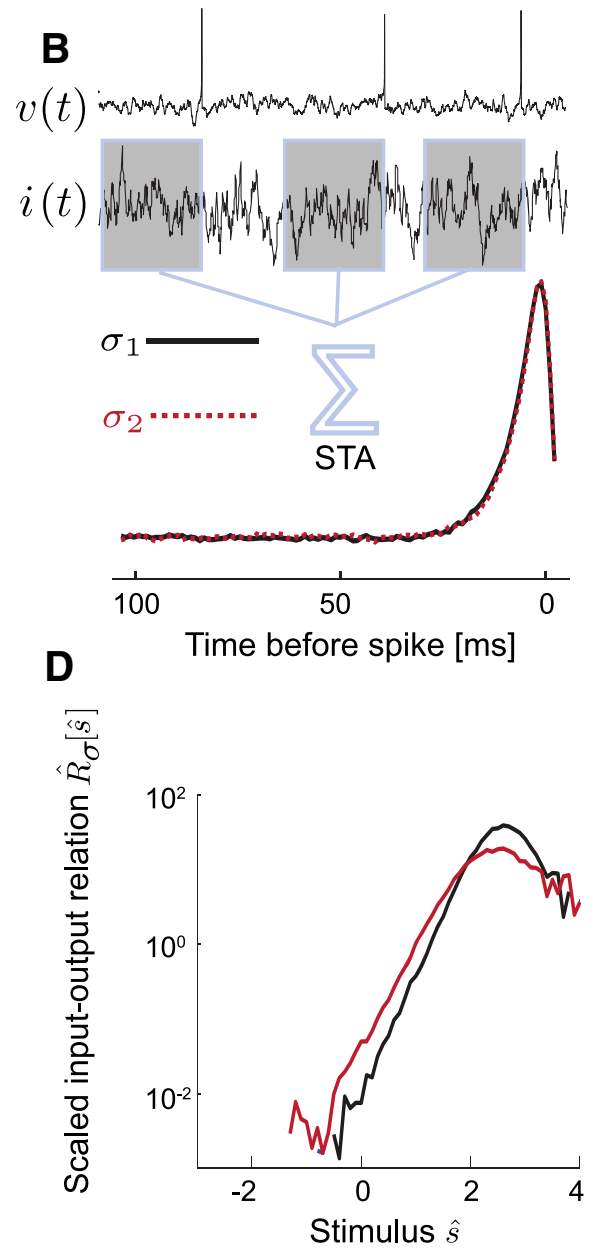

Figure 1. Characterizing the computation in single neurons with an $\mathrm{LN}$ model. $\boldsymbol{A}$, A neuron is driven to fire action potentials (in voltage $V(t)$ ) by stimulating with Gaussian noise input current $i(t)$. Increasing the SD, $\sigma$, of the input from $\sigma_{1}$ (top, black) to $\sigma_{2}$ (bottom, red) results in higher frequency firing. $\boldsymbol{B}$, The optimal single input feature correlated with spiking is the STA stimulus, the

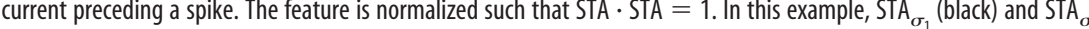
$p_{\sigma}[\hat{s} \mid \mathrm{sp}](\hat{s} \equiv s / \sigma)$. This neuron shows large error in gain scaling as the distribution changes shape significantly with changes is a unit variance Gaussian (shaded). $D$, Scaled nonlinear input- output relations, $\hat{R}_{\sigma}[\hat{s}] \equiv R_{\sigma}[\hat{s}] \bar{R}_{\sigma}$, are calculated by dividing $p_{\sigma}[\hat{s} \mid \mathrm{sp}]$ by $p[\hat{s}]$ (see Materials and Methods); as in $\boldsymbol{C}$, the two input-output relations do not overlap for different $\sigma$.

derlie the observed improvement of gain scaling in cortical neurons as they mature in the first postnatal week.

\section{Emergence of gain scaling does not require spontaneous activity}

Certain aspects of the electrophysiological development of cortical neurons, in particular those that lead to termination of spontaneous waves of activity (McCabe et al., 2006), depend on electrical activity. We therefore tested the hypothesis that spontaneous, synchronous network activity centered around P0 may trigger activity-dependent developmental events that impact gain scaling. From organotypic cultures, we compared the LN models of mature neurons cultured with and without TTX block of spontaneous activity from E18-P3 (Fig. 4). We found no significant difference between the TTX-treated and control STAs, input-output relations, and $I_{\mathrm{Na}} / I_{\mathrm{K}}$ ratios, indicating that the developmental acquisition of the common scaling input-output relation does not require a preceding period of spontaneous activity. We did not test responses to a range of gains for the control and TTX-cultured neurons, as the lack of difference in basic LN characterization and 
A

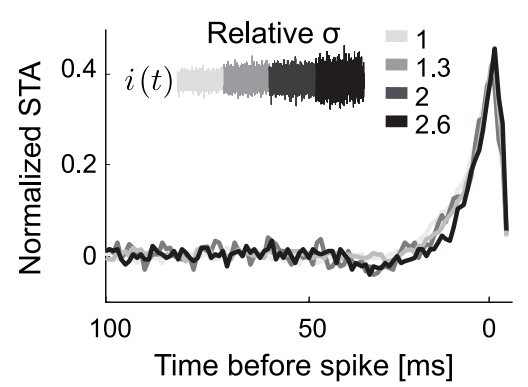

B

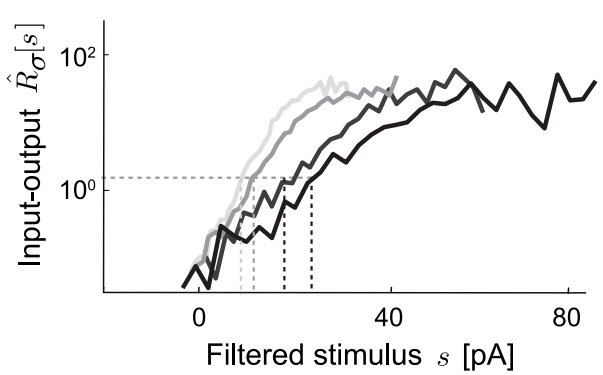

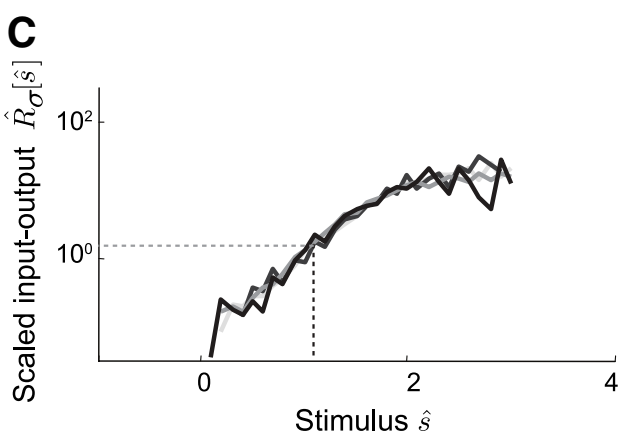

Figure 2. Gain scaling in single cortical neurons. $A$, Normalized STA current stimulus, P7 mouse cortical neuron. Separate STAs were calculated from four different stimulus SDs (relative $\sigma=\{1$, $1.3,2,2.6\}$; firing rates $\bar{R}_{\sigma}=\{5.1,6.9,7.5,10.0\} \mathrm{Hz}$ and spike counts $\left.n=\{1900,2600,600,800\}\right) . B$, Unscaled input- output relations, $R_{\sigma}[s] / \bar{R}_{\sigma}$, for a P7 mouse cortical neuron calculated for different input $\sigma$. Shading is the same as in $\boldsymbol{A}$. Dashed lines indicate the stimulus value at half-maximum. $\boldsymbol{C}$, The same input-output relations as in $\boldsymbol{B}$, scaled: $x$-axis $\hat{s} \equiv s / \sigma, y$-axis $\hat{R} \sigma[\hat{s}]$. This neuron shows nearly perfect gain scaling.
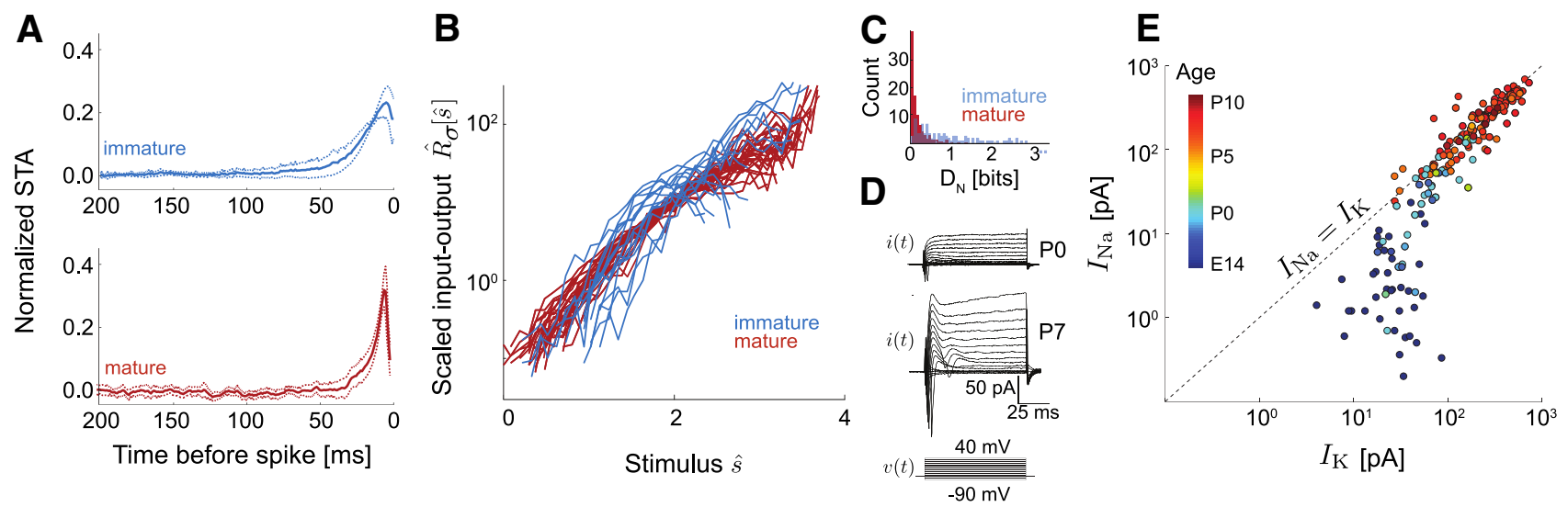

Figure 3. Convergence to a common intrinsic computation parallels development of voltage-gated currents. $\boldsymbol{A}$, Population STAs for immature and mature neurons. $\boldsymbol{B}, \hat{R}_{\sigma}[\hat{s}]$ for single $\sigma$ conditions for immature $\left(n=15\right.$, blue) and mature $\left(n=26\right.$, red) groups. C, Distribution of pairwise $D_{N}$ for immature and mature neurons shown in $A$ and $B$. Input- output relation shape is more consistent for mature $\left(\left\langle D_{N}\right\rangle=0.15 \pm 0.01\right.$ bits, $\left.n=325\right)$ than for immature $\left(\left\langle D_{N}\right\rangle=0.84 \pm 0.08\right.$ bits, $\left.n=120\right)$ neurons. $D$, Voltage-clamp protocol to measure maximal in vitro currents, $I_{\mathrm{Na}}$ and $I_{K}$, in immature (top) and mature (bottom) neurons. Steps start at a holding potential of $-70 \mathrm{mV}$ and range from -90 to $40 \mathrm{mV}$. $E, I_{\mathrm{Na}}$ versus $I_{\mathrm{K}}$ measured as in $E$. Warmer colors indicate increasing age $(\mathrm{E} 14-\mathrm{P} 11, n=169)$.

channel densities suggested that each group's intrinsic computation was the same with respect to the aspects we describe here.

\section{Gain-scaling behavior can be altered by pharmacological manipulation of $I_{\mathrm{Na}} / I_{\mathrm{K}}$}

An alternate explanation for the emergence of the scaling inputoutput relation is that immature neurons lack some additional intrinsic mechanism expressed by mature neurons, as there are many examples of specialized currents tuning the computational capabilities of single neurons (for review, see Bean (2007)). One possible mechanism was a significant 4 -AP-sensitive transient potassium current we observed only in mature neurons (Fig. 5). The characteristics of this current suggested that it could influence excitability at subthreshold voltages before a spike, possibly contributing to the input-output relation consistency and scaling observed in mature neurons. To test this hypothesis, we compared LN models from mature neurons with and without the addition of 4-AP. Surprisingly, for the majority of neurons, reducing the transient potassium current reversibly improved gain scaling (Fig. 5B) and resulted in a small increase in input-output relation similarity between neurons. Additionally, for a given $\sigma$, the input-output relation showed a decrease in slope and a shift toward smaller stimulus values (Fig. 5A). These effects are consistent with an increase in $I_{\mathrm{Na}} / I_{\mathrm{K}}$ and suggest that the effects of this transient K-conductance on gain scaling reflect its influence on the effective subthreshold current ratio.

These results, along with the age-dependent changes in inputoutput relations, predict that decreasing $I_{\mathrm{Na}} / I_{\mathrm{K}}$ in vitro should shift input-output relations to higher filtered stimulus values and decrease gain-scaling ability between stimuli with different distributions. To test these predictions, we determined inputoutput relations for in vitro neurons with a partial (5 nM) TTX block of sodium channels to reduce $I_{\mathrm{Na}} / I_{\mathrm{K}}$. At this TTX concentration, neurons still produced regular action potentials but at lower rates. As shown in Figure 5C, for a given stimulus distribution, application of TTX shifted the population mean inputoutput relation to a higher threshold stimulus value for firing (i.e., a translation along $\hat{s} \equiv s / \sigma$ ) relative to the control condition. We next compared gain scaling before and after application of TTX (Fig. 5D). For a gain contrast of $\Delta \sigma=30 \%$, the increase in error was not statistically significant. However, for $\Delta s=50 \%$, scaling was reversibly disrupted and $D_{\sigma}$ increased for all neurons by approximately fivefold. By demonstrating that gain scaling can be changed by altering sodium channel availability, these results support our hypothesis that the age-dependent increase in $I_{\mathrm{Na}} / I_{\mathrm{K}}$ is responsible for the emergence of gain scaling with development. 


\section{Gain scaling in a biophysical} neuron model

In combination, these in vitro data show that several remarkable properties of cortical neurons emerge in concert within the first postnatal week: neurons develop the ability to scale response gain to the amplitude of input fluctuations, they converge on a common population input-output relation, and they converge on a constant $I_{\mathrm{Na}} / I_{\mathrm{K}}$ ratio. To determine whether the observed developmental change in $I_{\mathrm{Na}} / I_{\mathrm{K}}$ ratio was sufficient to explain the emergence of the common scaling input-output relation, we extended the in vitro experiments described above by simulating a simple model of spike initiation in cortical neurons (Mainen et al., 1995), using only the active sodium and potassium currents responsible for spike generation. To recreate the spectrum of intrinsic properties we observed in developing cortical neurons, we determined LN models and investigated gain scaling over a grid of maximal sodium $\left(G_{\mathrm{Na}}\right)$ and potassium $\left(G_{\mathrm{K}}\right)$ conductances that spanned a range of $G_{\mathrm{Na}} / G_{\mathrm{K}}$ values (Fig. $6 B$ ). (Note that as the maximal conductance ratio is proportional to the maximal current ratio, we report conductance ratios here for convenience). Model neurons were classified as spontaneously active, excitable, or silent; these categories were separated by boundaries in the space of $G_{\mathrm{Na}}$ and $G_{\mathrm{K}}$ corresponding to particular values of the conductance ratio (Fig. 6A). LN characterizations were restricted to the excitable subset of model neurons $\left(G_{\mathrm{Na}} / G_{\mathrm{K}}\right.$ between 0.5 and 2).

We first examined how changes in model $G_{\mathrm{Na}} / G_{\mathrm{K}}$ determined input-output relation shape for a fixed stimulus distribution, as shown in the in vitro data (Fig. $3 B, C$ ). Figure $7 A$ overlays the scaled input-output relations $\hat{R}_{\sigma}[\hat{s}]$ for a range of $G_{\mathrm{Na}}$ and $G_{\mathrm{K}}$ combinations (inset), which gave rise to excitable models. For a given $\sigma, \mathrm{G}_{\mathrm{Na}} / G_{\mathrm{K}}$ determined the shape of the scaled input-output relation. As the conductance ratio was increased, the input-output relations decreased in slope until they converged to a fixed shape for large $G_{\mathrm{Na}} / G_{\mathrm{K}}$. For low $G_{\mathrm{Na}} / G_{\mathrm{K}}$ models, input-output relations were quite variable compared with high ratio models, consistent with the finding that immature neurons show variable input-output relations, while mature neurons display a common population inputoutput relation in step with the developmental increase in maximal $I_{\mathrm{Na}} / I_{\mathrm{K}}$.

We next compared the models' gain-scaling capabilities by stimulating firing with noise of different SDs (Fig. 7B). Models with low $G_{\mathrm{Na}} / G_{\mathrm{K}}$ had input-output relations, which did not scale completely with $\sigma$, while those input-output relations from high $G_{\mathrm{Na}} / G_{\mathrm{K}}$ models were nearly identical for all $\sigma$. We extended the range of $\sigma$ beyond what was possible in vitro and compared gainscaling ability across different changes in $\sigma$ and values of $G_{\mathrm{Na}} / G_{\mathrm{K}}$ (Fig. 7C). Scaling was highly correlated with increasing ratio, rather than either conductance alone (Fig. 6B), and, for those $=1.18 \pm 0.20, p=0.09$ ).
B
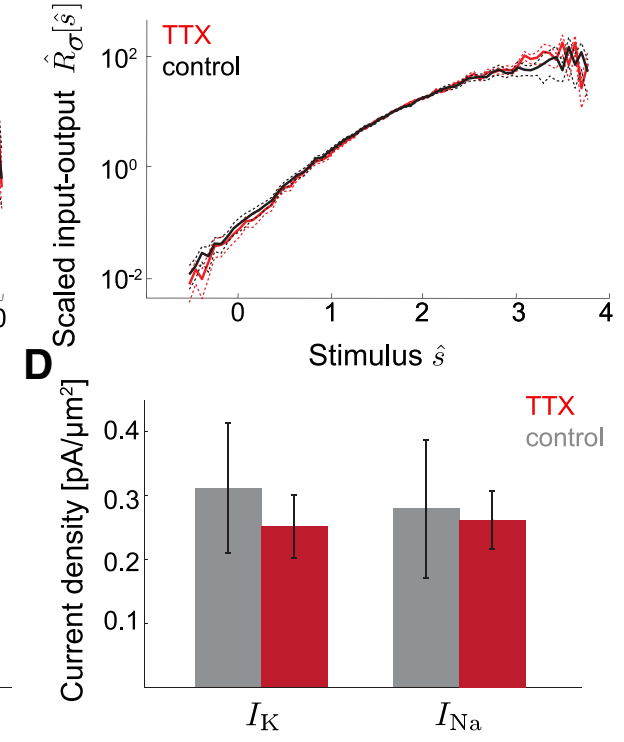

Figure 4. Block of spontaneous activity does not significantly affect intrinsic computation. Single input $\sigma \mathrm{LN}$ models were culated for P7 neurons cultured with (red, $n=13$ ) or without (black, $n=13$ ) TTX block of spontaneous activity from E17 to P3. 列 ratios were not significantly different between conditions $\left(I_{\mathrm{K}}: p=0.58 ; I_{\mathrm{Na}}: p=0.88 ;\right.$ control $\left\langle I_{\mathrm{Na}} / I_{\mathrm{K}}\right\rangle=0.90 \pm 0.06 ; \operatorname{TTX}\left\langle I_{\mathrm{Na}} / I_{\mathrm{K}}\right\rangle$

models with incomplete gain scaling, the input-output relation mismatch $D_{\sigma}$ increased for larger changes in $\sigma$ (Fig. $7 C$ ). The model scaling performance was similar to the in vitro $I_{\mathrm{Na}} / I_{\mathrm{K}^{-}}$ dependent developmental increase in scaling ability. Thus, in vitro and in the model, the most accurate gain scaling occurs with a common input-output relation, which is determined by the ratio of spike-generating currents and converges to a common form for large $I_{\mathrm{Na}} / I_{\mathrm{K}}$.

We also observed a decrease in the intrinsic integration properties of the neurons during development (Figure $3 A$ ). This change is mostly likely due to the known decrease in input resistance and concomitant shortening of membrane time constant, which occurs over the first postnatal week (Picken-Bahrey and Moody, 2003a; Moody and Bosma, 2005). We also explored the effects of input resistance on gain scaling in the model, but while the width of the STA was increased with decreased leak, gain scaling remained the same for this manipulation (data not shown).

\section{A simple neuron model reproduces the gain scaling observed in mature neurons}

The biophysical model demonstrates that perfect gain scaling in the input-output relation can occur without any explicit adaptive processes that adjust the gain through spike-driven negative feedback, such as slow sodium inactivation (Kim and Rieke, 2001; Lundstrom et al., 2010) and slow afterhyperpolarization (AHP) currents (Ermentrout, 1998; Lundstrom et al., 2010). However, to understand the mechanism for this adaptive computation for some parameter choices, we would 

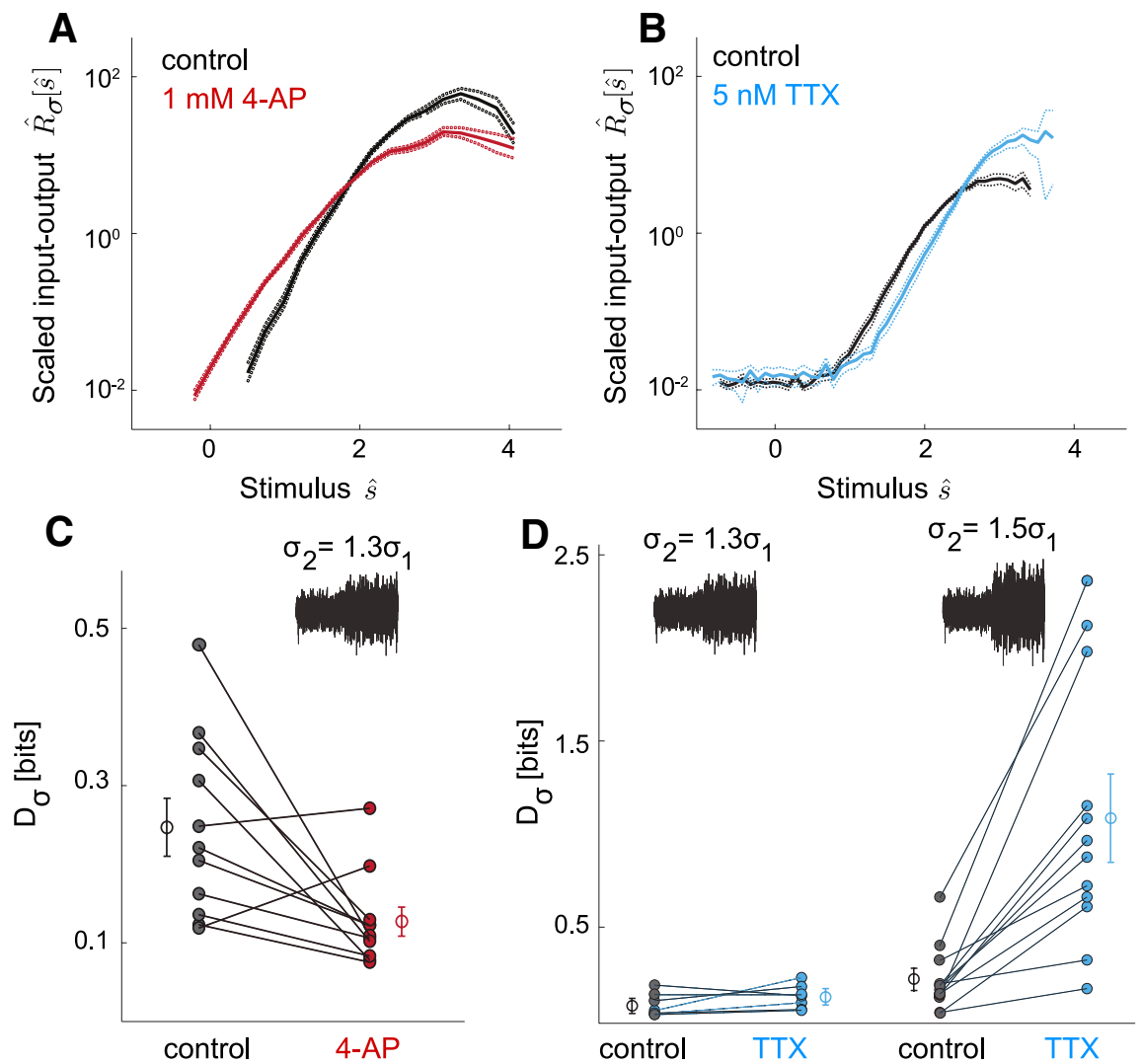

Figure 5. Two pharmacological manipulations of $I_{\mathrm{Na}} / I_{\mathrm{K}}$ change gain-scaling behavior in agreement with model results $\boldsymbol{A}, \boldsymbol{B}$, Reduction of transient K-current changes input- output relation shape and improves gain scaling (organotypic slices). $\boldsymbol{A}$, Mean $\hat{R}_{\sigma}[\hat{s}]$ across neurons before (black) and after the addition of $1 \mathrm{~mm} 4-\mathrm{AP}(\mathrm{red}), n=11$. Dashed lines show $\pm \mathrm{SEM}$. $\boldsymbol{B}$, Mean $D_{\sigma}$ values before (black) and after addition of 4-AP (red). Lines show control and 4-AP pairings for individual neurons (control: $\left\langle D_{\sigma}\right\rangle=0.27 \pm 0.03$ bits; $4-A P:\left\langle D_{\sigma}\right\rangle=0.16 \pm 0.01$ bits, $n=11$ ). A majority of neurons showed an improvement in gain scaling ( 9 of 11 neurons, $p=0.014$, paired $t$ test, mean improvement of $35 \pm 8 \%$ ). Treatment with 4 -AP resulted in a small increase in input- output relation similarity, $D_{\mathrm{N}}(p=0.013) . \boldsymbol{C}, \boldsymbol{D}$, Partial block of sodium channels shifts the input-output relation shape to higher stimulus values and decreases gain-scaling behavior (acute slices). $\boldsymbol{C}$ Mean $\hat{R}_{\sigma}[\hat{s}]$ across neurons before (black) and after the addition of 5 nм TTX (blue), $n=19$. Dashed lines show \pm SEM. Stimulus SD were changed by a factor $\Delta \sigma=100\left(\sigma_{2}-\sigma_{1}\right) / \sigma_{1} . D$, Mean $D_{\sigma}$ values before (black) and after addition of 5 nм TTX (blue) for $30 \% \Delta \sigma$ (left) and $50 \% \Delta \sigma$ (right) switches. Note change in abscissa scale from $\boldsymbol{B}$. Lines show control and TTX pairings for individual neurons; $30 \% \Delta \sigma(n=7)$ (control: $\left\langle D_{\sigma}\right\rangle=0.08 \pm 0.02$ bits; TTX: $\left\langle D_{\sigma}\right\rangle=0.13 \pm 0.03$ bits). A majority (5 of 7 neurons) showed an increase in gain-scaling error, but this difference was not statistically significant $(p=0.1773$, paired $t$ test; mean increase in error of $99 \pm 52 \%) ; 50 \% \Delta \sigma(n=12)$ (control: $\left\langle D_{\sigma}\right\rangle=0.21 \pm 0.05$ bits; TTX: $\left\langle D_{\sigma}\right\rangle=1.09 \pm 0.21$ bits). For this $\Delta \sigma$, all neurons showed an increase in gain-scaling error $(p=0.0004$, paired $t$ test; mean increase in error of $550 \pm 150 \%)$. Error bars indicate SEM.

like to simplify the neuron's dynamics further. We use the EIF model (Fourcaud-Trocmé et al., 2003), which is sufficiently rich to quantitatively fit recorded data but is also simple enough to analyze theoretically.

The EIF model (Eq. 15) is specified by seven parameters. The effective resting potential, $v_{o}$, is the steady-state voltage for fixed input mean $\mu$. The threshold voltage, $v_{t h}$, defines the separation between subthreshold and spiking dynamics. Together, $v_{t h}-v_{o}$ sets the distance-to-threshold-the typical voltage scale that governs the response of the neuron. The activation parameter, $\Delta$, sets the voltage scale over which sodium activates near threshold; the reset voltage, $v_{r}$, approximates spike afterpolarization; and $v_{s}$ sets the maximum voltage at the top of the spike. It is convenient to express the input $\mathrm{SD}$ in terms of the scale of the corresponding voltage fluctuations, independent of the membrane time constant, $\tau_{\mathrm{v}}$, and the total membrane area; we denote this input parameter $\sigma_{\mathrm{v}}$ (Eq. 2). The complete behavior of the EIF model may be character- ized by the three dimensionless ratios describing spike initiation $\Delta /\left(v_{t h}-v_{o}\right)$, afterpolarization $\left(v_{r}-v_{o}\right) /\left(v_{t h}-v_{o}\right)$, and the relative input strength $\left(\sigma_{v} /\left(v_{t h}-v_{o}\right)\right.$. For the neurons studied here, in which spike-generating kinetics are fast compared with the membrane time constant, the maximal conductance ratio, $G_{\mathrm{Na}} / G_{\mathrm{K}}$, can be related with an inequality to the distance from rest to threshold, $v_{t h}-v_{o}$, as shown by Equation 20 in Materials and Methods:

$$
\frac{\partial\left(v_{t h}-v_{o}\right)}{\partial\left(\frac{G_{\mathrm{Na}}}{G_{\mathrm{K}}}\right)}<0
$$

Thus, increasing the biophysical conductance ratio corresponds to decreasing the distance-to-threshold in a comparable EIF model.

The EIF model gives a good fit to the mature cells (Fig. 8). For each cell (P7, $n=6$ ), we identified a set of parameters that best generalized across all input SDs studied $\left(0.25 \leq \frac{\sigma_{\mathrm{v}}}{v_{\text {th }}-v_{o}} \leq 0.89\right)$. The EIF fits reproduce the voltage traces in detail and correctly predict the inputoutput relations of the data (Figure 10). The best-fit parameters for mature neurons are summarized in Table 2.

LN models obtained for the EIF model also reproduce the observations seen in the biophysical model. With fixed model kinetics (fixed $\Delta$ and $v_{r}$ ), the input-output function of the EIF models converge to a common form as the distance-to-threshold,

$v_{t h}-v_{o}$, decreases. Furthermore, decreasing distance-to-threshold correlates with improved gain scaling and less steeply sloped input-output curves. As decreasing distance-to-threshold is equivalent to increasing the maximal conductance ratio, the EIF model behavior is consistent with our observations that gain scaling occurs for large conductance and large maximal current ratios seen in mature neurons. To see the correspondence of changes of $v_{t h}-v_{o}$ in the EIF model, compare Figure 10 to Figure 7 for changes of $G_{\mathrm{Na}} / G_{\mathrm{K}}$ in the biophysical model; Figure 5 for pharmacological manipulations involving TTX and 4-AP; and Figure 3 for maturation and maximal current expression.

\section{Relating gain scaling to voltage dynamics in simplified neurons}

To understand the basis for the observed gain scaling, we need to determine the relationship of the input-output relation of the LN model to the voltage-based dynamics that support gain scaling. Here we argue that the gain scaling we observe can be understood in terms of the subthreshold voltage distribution with different input SDs. We derive the properties of the subthreshold distribution for neurons which perform gain scaling, and show 
that these properties hold for LIF neurons in the limit of large noise input. EIF neurons also possess these properties but with biologically plausible neuronal parameters. Finally, the experimental data also has the same properties.

The neuronal dynamics implement the LN model computation by encoding the filtered stimulus in the membrane voltage: the filtering properties of the membrane, modulated by feedback from the AHP, select from the input current $i(t)$ a specific component, $s(t)$, which is best correlated with spiking. The input-output relation follows from the conditional voltage distribution evaluated at the spike threshold, $v_{\text {spike }}$ :

$$
R_{\sigma}[s] \propto \sigma p_{\sigma}\left[v_{\text {spike }} \mid s\right]
$$

where $p_{\sigma}\left[v_{\text {spike }} \mid s\right]$ is a function of $s$ for fixed $v_{\text {spike }}$, for a given $\sigma$. The factor of $\sigma$ in Equation 23 arises because the probability of crossing threshold is proportional to the typical size of a voltage step that occurs in a single time step; the proportionality constant depends on the precise definition of $v_{\text {spike }}$ (see Materials and Methods for discussion) and the correlation time of the input (Famulare and Fairhall, 2011). In terms of the scaled input-output relation $\hat{R}(\hat{\sigma})$, this becomes the following:

$$
\hat{R}_{\sigma}[\hat{s}] \propto \frac{\sigma p_{\sigma}\left[v_{\text {spike }} \mid \hat{s}\right]}{\bar{R}_{\sigma}}
$$

where $\bar{R}_{\sigma}$ is the mean firing rate.

Perfect gain scaling requires that the input-output relations for different $\sigma$ are equal when the stimulus is scaled by $\sigma$, $\bar{R}_{\sigma_{1}}[\hat{s}]=\bar{R}_{\sigma_{2}}[\hat{s}]$. Averaging over $\hat{s}$ shows that this relationship can be satisfied when the probability density evaluated at $v_{\text {spike }}$ is a constant, independent of $\sigma$,

$$
p_{\sigma}\left[v_{\text {spike }}\right]=\text { constant; }
$$

and the mean firing rate is proportional to $\sigma$,

$$
\bar{R}_{\sigma} \propto \sigma
$$

For an LIF neuron, the derived input-output relations display gain scaling in the limit that the scale of the voltage fluctuations driven by the input noise is very large compared with the intrinsic voltage scale, $v_{t h}-v_{o}$ (note that this is the EIF model in the limit of $\Delta \rightarrow 0$ ) (Famulare and Fairhall, 2011). In this limit, the dynamics of the neuron are linear below threshold with a "reflective barrier" (Risken, 1996) formed by the rapid spike-and-reset (Fig. $11 B$ ). In this case, the rate scales with $\sigma$ (Fourcaud and Brunel, 2002; Famulare and Fairhall, 2011):

$$
\bar{R}_{\sigma \rightarrow \infty}=\frac{\sigma}{\sqrt{\pi}\left(v_{t h}-v_{r}\right) \tau_{V}},
$$

One can also show that with short but finite input correlation time $\tau_{\mathrm{c}}$, at $v_{t h}, p_{\sigma}\left[v_{t h}\right]$ becomes a constant independent of $\sigma$, $\frac{1}{v_{t h}-v_{r}} \sqrt{\frac{\tau_{\mathrm{c}}}{\pi \tau_{v}}}$. Furthermore, the steady-state voltage distribution, Equation 21, is close to a truncated Gaussian with a width that scales with $\sigma$ (Fig. 11B) (Famulare and Fairhall, 2011):

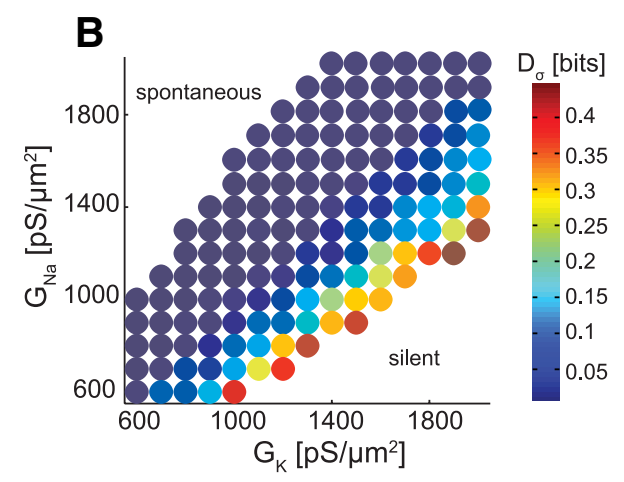

Figure 6. Excitability and gain-scaling error in the $G_{\mathrm{Na}}$ versus $G_{\mathrm{K}}$ conductance plane. $\boldsymbol{A}$, The biophysical model had three citability regimes: silent, excitable, and spontaneously active (10,000 simulations, $G_{\mathrm{Na}}$ and $G_{K}$ from 20 to $2000 \mathrm{pS} / \mu \mathrm{m}^{2}$, in $\mathrm{pS} / \mu \mathrm{m}^{2}$, in increments of $\left.100 \mathrm{pS} / \mu \mathrm{m}^{2}\right) \cdot \boldsymbol{B}, D_{\sigma}$ was calculated for 148 model neurons with varying $G_{\mathrm{Na}}$ and $G_{\mathrm{K}}$ conductance values, $\sigma_{2}=1.3(\Delta \sigma=30 \%)$. Warmer colors indicate larger gain-scaling error

$$
p_{\sigma \rightarrow \infty}[v]= \begin{cases}\frac{2}{\sqrt{\pi} \sigma_{\mathrm{v}}} e^{-\frac{v^{2}-v_{r}^{2}}{\sigma_{\mathrm{v}}^{2}}}, & \text { if } v \leq v_{r} \\ \frac{2}{\sqrt{\pi} \sigma} \frac{v_{t h}-v}{v_{t h}-v_{r}}, & \text { if } v_{r}<v<v_{t h}\end{cases}
$$

for small input correlation time. Thus, not only the value of the density at threshold is constant, the entire subthreshold distribution scales such that $p_{\sigma}[v]$ obeys

$$
p_{\sigma}\left[\frac{v-v_{\text {spike }}}{\sigma}\right] \text { is independent of } \sigma
$$

Therefore, for the LIF model, the rate indeed scales with $\sigma$ and the subthreshold voltage distribution $p[v]$ is independent of $\sigma$.

Although derived for the LIF model, these conditions depend only on model-independent quantities, $p_{\sigma}[v], v_{\text {spike }}$, and $\bar{R}_{\sigma}$. Therefore, we investigated whether the same conditions hold for the EIF neuron for less extreme input amplitudes, which are more biologically plausible. The EIF model does indeed obey these conditions approximately for finite values of $\left(v_{t h}-v_{o}\right)$ and sufficiently large $\sigma$ (Famulare and Fairhall, 2011); this is shown in Figures 9 and $11 C$. The subthreshold distribution for EIF neurons is distorted from the LIF solution by the subthreshold nonlinearities responsible for spike initiation and the postspike repolarization (Fig. 11C) (see Materials and Methods). However, when the effect of the subthreshold nonlinearity is weak, the distribution well below threshold remains close to the Gaussian distribution of a linear membrane and the distribution above threshold remains approximately independent of $\sigma$ (Figure 9). With these conditions on the distribution satisfied, gain scaling occurs for EIF models for which the distance from rest to threshold is biologically plausible (Figs. 9, 10).

Finally, we also examine these quantities experimentally. For the P7 cells that exhibit nearly perfect gain scaling, Equations 25 and 29 are satisfied in the gain-scaling regime (Fig. 11D). A test of the proportionality of the mean firing rate to the input SD (Eq. 26 ), is shown both for the EIF model and the experimental data for mature cells in Figure $8 A-C$. The degree of gain scaling correlates with the linear dependence on $\sigma$ of the mean firing rate.

Because of the strong dependence of gain scaling on the distance-to-threshold $v_{t h}-v_{o}$, its existence in these neurons required a depolarizing mean current to drive the effective resting 

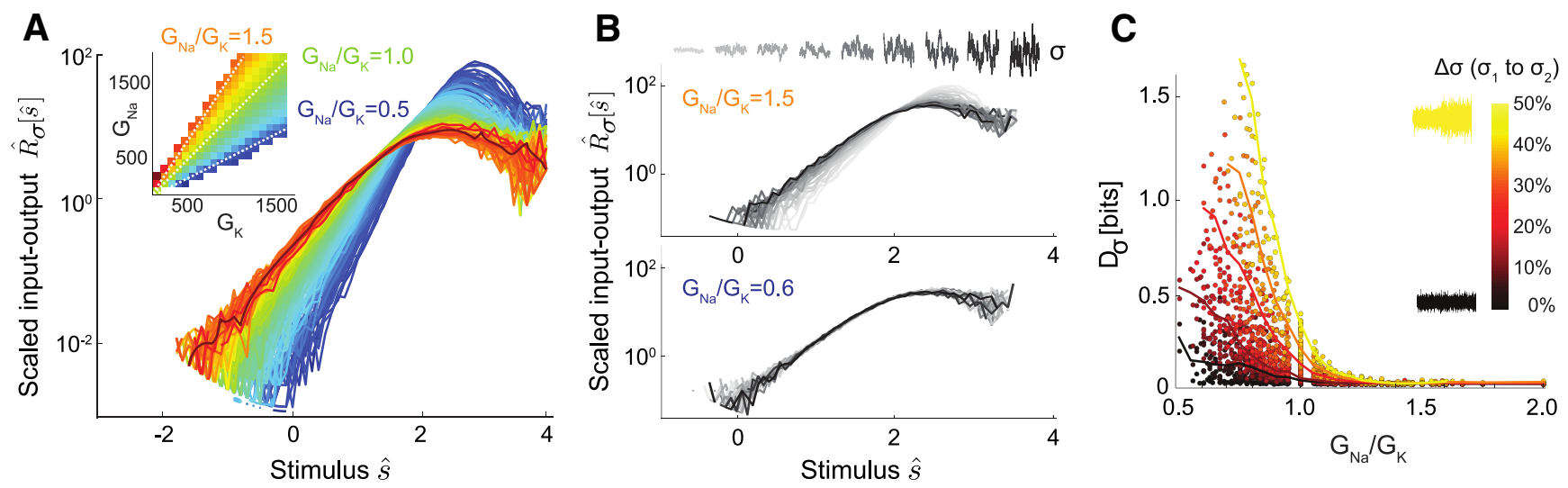

Figure 7. Conductance ratio changes input- output relation shape and gain-scaling ability. $A, \hat{R}_{\sigma}[\hat{s}]$ for model neurons with a variety of conductance ratios $\left(G_{\mathrm{Na}} / G_{K}\right.$, see inset for color code), stimulated with a single stimulus $\sigma(70 \mathrm{pA}) \cdot \boldsymbol{B}, \hat{R}_{\sigma}[\hat{s}]$ for a range of $\sigma(50-100 \mathrm{pA})$ for two model neurons with high and low $G_{\mathrm{Na}} / G_{\mathrm{K}} \cdot C$, Error in rescaling, $D_{\sigma^{\prime}}$ plotted against $G_{\mathrm{Na}} / G_{\mathrm{K}}$ for models stimulated with a large range of $\sigma . D_{\sigma}$ was calculated for conductance combinations, which responded to both $\sigma_{1}$ and $\sigma_{2}$ f for $\Delta \sigma$ from 5 to $50 \%$. Lines indicate mean $D_{\sigma}$ values for different levels of $\Delta \sigma$.

A
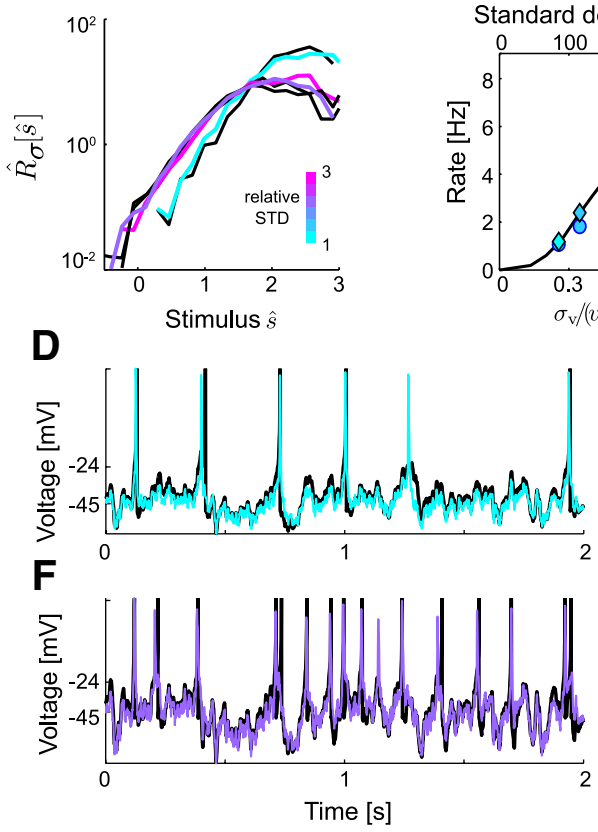

B

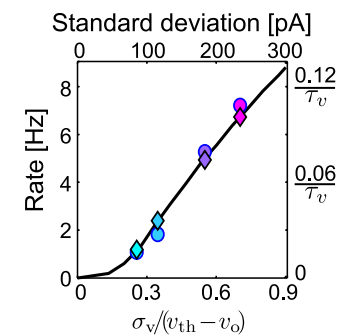

$\mathbf{E}$
C

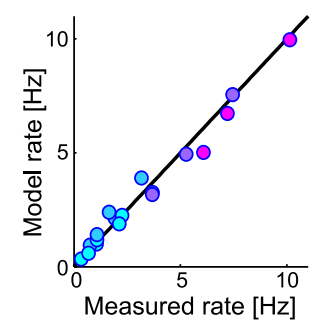

Bahrey and Moody, 2003a; McCabe et al., 2006). These depolarizations last for at least hundreds of milliseconds and move the baseline voltage into the range of -50 to $-40 \mathrm{mV}$, around which synaptic inputs can cause spikes (Picken-Bahrey and Moody, 2003a). Thus, the gain-scaling property observed here is likely modulated by and interacts with network activity.

Estimate of the magnitude of the maximal current ratio $I_{\mathrm{Na}} / I_{\mathrm{K}}$, for mature neurons

We estimate the maximal current ratio, $I_{\mathrm{Na}} / I_{\mathrm{K}}$, from the EIF model fits for mature cells. The size of the maximal $I_{\mathrm{K}}$ is measured in steady-state at $+40 \mathrm{mV}$, where the potassium channels are assumed to be maximally open. In terms of the biophysical model in Equation 8, we have $I_{\mathrm{K}} \approx$ $G_{\mathrm{K}}\left(40-E_{\mathrm{K}}\right)$ assuming $n(40) \approx 1$. In the mature cells, where the maximal potassium current is sufficiently large such that the potassium current dominates the spike AHP, the maximal potassium current can be related to $v_{r}-v_{o}$ in the EIF model. In order of magnitude, the change in voltage during the AHP is determined by the mean potassium current during the AHP relative to the baseline potassium current away from spikes: $v_{r}-v_{o} \sim r$ $\left(\left\langle i_{\mathrm{K}}\right\rangle_{\mathrm{AHP}}-\left\langle i_{\mathrm{K}}\right\rangle_{o}\right)$, where $r$ is the membrane resistance near $v_{o}$. The mean current during the AHP is approximately: $\left\langle i_{\mathrm{K}}\right\rangle_{\mathrm{AHP}} \approx$ $G_{\mathrm{K}}\langle n\rangle_{\mathrm{AHP}}\left(E_{\mathrm{K}}-\langle v\rangle_{\mathrm{AHP}}\right)$. During the AHP, $\langle n\rangle_{\mathrm{AHP}} \sim 1 / 2$ generically since the channels go from more open to more closed

potential to $v_{o}>-50 \mathrm{mV}$, whereas the true resting potentials were approximately $-68 \mathrm{mV}$ in mature cells (Table 2). Without the depolarizing current, gain scaling could not occur because the distance to threshold would be far too large to allow gain scaling with biologically realistic input strengths. When network synaptic activity is not blocked, such a net depolarizing current exists in vitro in the form of large spontaneous depolarizations (Picken- states, and $\langle v\rangle_{\mathrm{AHP}} \sim v_{0}+v_{r}-v_{o} / 2 \sim-50 \mathrm{mV}$ (Table 2). The magnitude of $\left\langle i_{\mathrm{K}}\right\rangle_{o}$ is generally much smaller than $\left\langle i_{\mathrm{K}}\right\rangle_{\mathrm{AHP}}$ because potassium currents are activated during the spike and $\langle v\rangle_{\mathrm{AHP}}-$ $E_{\mathrm{K}}>v_{o}-E_{\mathrm{K}}$. Finally, re-expressed in terms of the maximal current and using the reversal potentials reported in Table 1 from Mainen et al. (1995), we have the following: 


$$
\begin{aligned}
v_{0}-v_{r} & \sim r I_{\mathrm{K}} \frac{\left(\langle n\rangle_{\mathrm{AHP}}\langle v\rangle_{\mathrm{AHP}}-E_{\mathrm{K}}\right)}{\left(40-E_{\mathrm{K}}\right)}, \\
& \sim 0.1 r I_{\mathrm{K}}
\end{aligned}
$$

For the immature cells (E14-P0), the maximal potassium conductance is too small for the above analysis to be relevant: there is no pronounced AHP (PickenBahrey and Moody, 2003b). Rather, the leak is primarily responsible for the return to rest and refractory behavior is almost solely due to the inactivation of the small pool of sodium channels. Thus, for immature cells, there is no direct correspondence between the EIF model reset and the potassium current.

To determine the maximal sodium current, the voltage-clamp examples in Figure $3 D$ show that the maximum occurs for voltage steps from $-70 \mathrm{mV}$ to approximately the threshold voltage around -30 $\mathrm{mV}$ : the maximal sodium current is approximately the transient sodium current at threshold, where the sodium current transiently approaches maximum activation with minimal inactivation. Using Equation 19, the sodium current at threshold is related to $v_{t h}-v_{o}$ in the EIF model:

$$
v_{t h}-v_{o} \sim r I_{\mathrm{Na}},
$$

where we have assumed that sodium current at rest is small and that the potassium current is small compared with sodium during spike generation.

Together, the maximal current ratio is determined approximately by the size of the reset relative to the threshold. Using the mean bestfit EIF parameter ratio in Table 2, we estimate for the maximal current ratio for mature gain-scaling cells as follows:

$$
\frac{I_{\mathrm{Na}}}{I_{\mathrm{K}}} \sim \frac{0.1\left(v_{t h}-v_{o}\right)}{v_{o}-v_{r}}=1,
$$

as is consistent with the experimental observations.

\section{Discussion}

To characterize the developmental changes in single neuron computation in the sensorimotor cortex, we constructed functional LN models describing the encoding of current inputs with varying statistical properties in output spikes (Fig. 1). In the LN model framework, changes in a neuron's selectivity can appear either as changes in the spike-triggering feature, in the input-output relation, or both. While the shape of the STA can reflect stimulus statistics and statistical interactions between spikes (Theunissen et al., 2000; Agüera y Arcas et al., 2003; Pillow and Simoncelli, 2003; Yu and Lee, 2003; Paninski et al., 2004; Sharpee et al., 2004; Powers et al., 2005; Famulare and Fairhall, 2010, 2011; Atick, 2011), here we observed that the STA had a fixed form, indicating that the basic feature driving spiking was independent of stimulus statistics. However, we found that gain scaling - the maintenance of a constant scaled input-output relation shape across different stimulus distributions (Fig. 2) - to changes in the typical size of input fluctuations around a base-
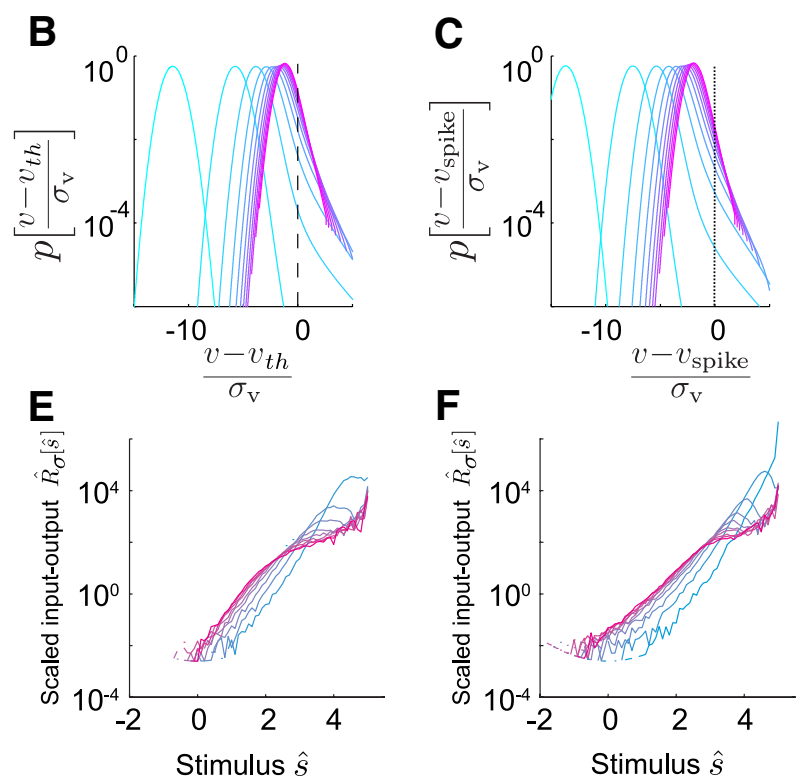

Figure 9. Gain-scaling properties of the EIF neuron. All parts correspond to the mean best-fit ElF neuron from Table 2; cool-to-warm color gradient corresponds to increasing input SD, $\sigma_{\mathrm{v}}$ from 2 to $24 \mathrm{mV}$. $\boldsymbol{A}$, Steady-state voltage distributions (unscaled); the dashed line indicates constant $v_{t h}$; the dotted line intersects each distribution at its $v_{\text {spike, }}$, the voltage for with less ambiguity and slightly more accurately captured the gain-scaling property of the model, as was indicated by the versus input SD. As is consistent with the scaling of the voltage distribution for increasing input $S D$, the firing rate 列 $\boldsymbol{B}$, computed using spike times triggered on crossing of $v_{t h} . \boldsymbol{F}$, Nonlinear functions as in $\boldsymbol{E}$, computed using spike times triggered on $v_{\text {spike, }}$, have a slightly different shape but very similar gain-scaling properties.

line appears during the first postnatal week of cortical development and is a function of age-dependent changes in the ratio of primary spike-generating currents, $I_{\mathrm{Na}} / I_{\mathrm{K}}$. Using a biophysical model and the simple EIF model, we then demonstrated that tuning only the spikegenerating currents is sufficient to replicate our main experimental findings. We used the reduced model to determine the necessary conditions under which a neuronal dynamical system will exhibit gain scaling. We showed that these are obeyed by the cortical neurons, and predicted the conditions when the property fails to hold.

Different electrophysiological classes of neurons begin to emerge during the first postnatal week as more diverse ion channel types are expressed (Connors and Gutnick, 1990; Massengill et al., 1997; Vacher et al., 2008); in particular, it is often potassium currents that sculpt feature selectivity (Bean, 2007). In our sample population, a transient potassium current was the most prominent current beyond $I_{\mathrm{Na}}$ and $I_{\mathrm{K}}$ and we initially expected that this current may be responsible for the precise gain-scaling capabilities of mature neurons. However, reducing this current improved gain scaling in the majority of neurons (Fig. 5). While the lengthy noise stimulation protocol we used here precluded the possibility of testing for a wide range of additional currents, in combination with our simulation results and the theory, these findings (Fig. 7) suggest that a similar effect would be seen for other currents that change the effective subthreshold $G_{\mathrm{Na}} / G_{\mathrm{K}}$ ratio. It is possible 

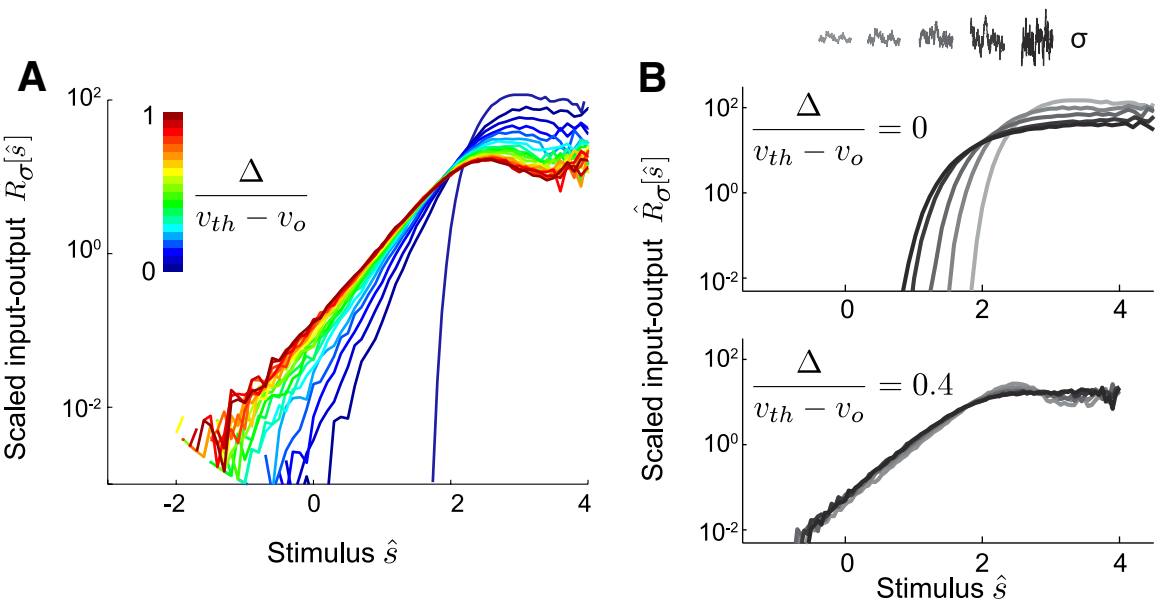

C

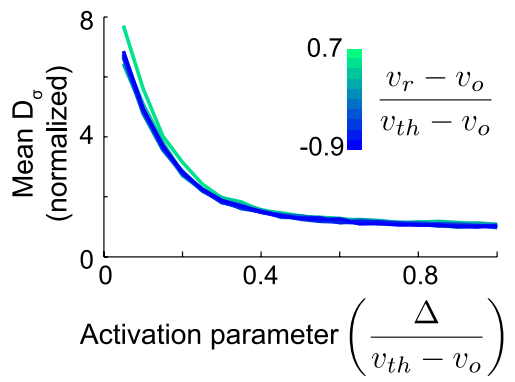

Figure 10. EIF model distance-to-threshold changes input-output relation shape and rescaling ability in agreement with biophysical modeling. $\boldsymbol{A}, \hat{R}_{\sigma}[\hat{s}]$ for the EIF model over a range of relative activation parameters $\left(0 \leq \Delta / v_{t h}-v_{o} \leq 1\right.$ in increments of $\left.0.05 ; v_{r}=v_{o}\right)$, stimulated with a single stimulus $\sigma_{v} / v_{t h}-v_{o}=0.65$. The gain (slope) decreases with increasing $\Delta / v_{t h}-v_{o} ;$ i.e., with decreasing distance to threshold, corresponding to increasing $G_{\mathrm{Na}} / G_{\mathrm{K}}$ (compare with Fig. $\left.7 A\right)$. $B, \hat{R}_{\sigma}[\hat{s}]$ for a range of input strengths $\left(0.65 \leq \sigma v / v_{\text {th }}-v_{0} \leq 2\right)$ for two model neurons with different $\Delta / v_{t h}-v_{o}$ (compare with Fig. $7 B$ ). $C$, Mean error in rescaling, $\left\langle D_{\sigma}\right\rangle$ (averaged over range of $\sigma_{v}$ in $\boldsymbol{B}$, normalized relative to the minimum value) plotted against the activation parameter (compare with Fig. 7C). For fixed $\Delta$, gain scaling improved with decreasing $v_{t h}-v_{o}$ and was insensitive to the reset parameter.

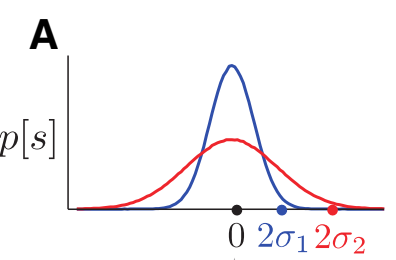

B
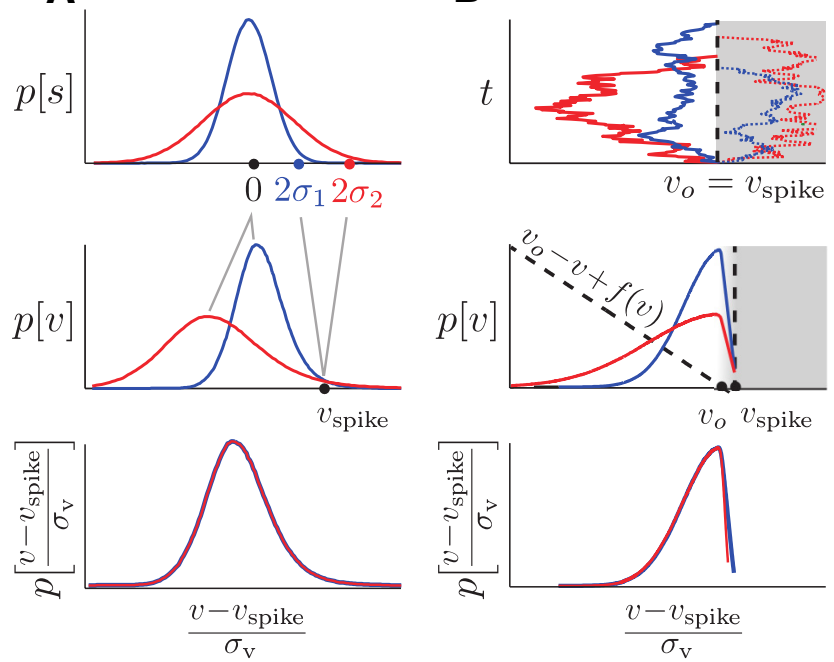
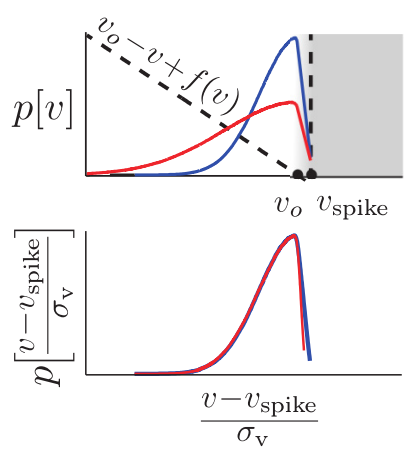

C
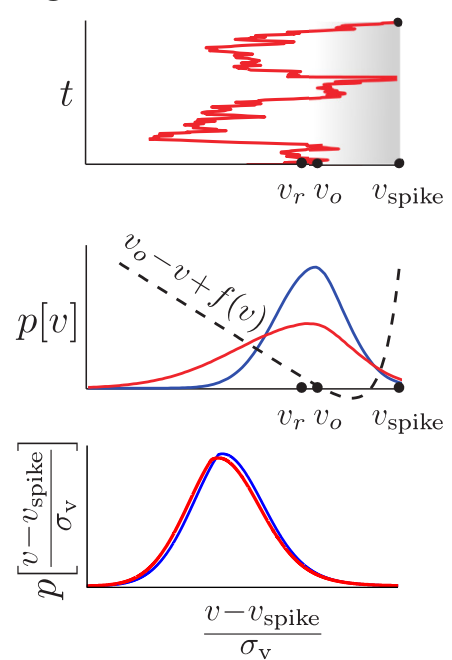

D
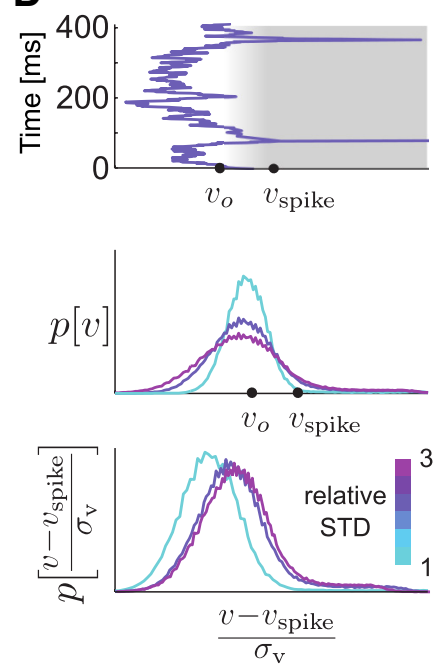

Figure 11. Computing with a voltage-based neuron: gain scaling. $\boldsymbol{A}$, Idealized example: perfect gain scaling is enacted by the transformation of the filtered stimulus into the voltage. Consider an example where a spike is triggered by a $2 \sigma$ event in the filtered stimulus (top). To perform perfect gain scaling, in stimulus space, the threshold for firing a spike must increase with increasing $\sigma$ so that the encoded event is determined by the relative size of the signal. The variable threshold in stimulus space maps to a fixed threshold in voltage space (middle) and so the mass of the voltage distribution must correspondingly shift to more hyperpolarized voltages to enact gain scaling. Gain scaling will be assured if the scaled, shifted voltage distribution, Equation 29 , is identical for all input $\sigma . \boldsymbol{B}$, Limiting dynamics: linear subthreshold integration with a voltage threshold very close to the resting potential. In this limit, all trajectories that start from rest and move to hyperpolarized voltages linearly integrate the input and so have typical displacement proportional to $\sigma_{v}$, while all depolarized trajectories are truncated by the occurrence of a spike (top; inaccessible region shaded). The voltage distribution is half-Gaussian except for the small region between rest and threshold (middle) and scales with $\sigma_{v}$ (bottom). In this limit, the system approximates a linear stochastic process with a reflecting boundary at the threshold. C, Gain scaling in the EIF model. Example voltage trace (top); steady-state voltage distribution with intrinsic dynamics overlaid (middle); scaled voltage distribution (bottom). The subthreshold behavior occurs primarily where the dynamics are essentially linear. The intrinsic excitability facilitates spiking, speeding the transit through the region near $v_{\text {spike }}$ and allowing for nearly perfect gain scaling in the presence of a large nonlinearity and distance to threshold. $\boldsymbol{D}$, Gain scaling in an example mature cortical neuron. Voltage trajectory (top): as in the EIF model, input integration occurs primarily below the effective resting potential; steady-state voltage distributions (middle); scaled voltage distributions (bottom). For larger input SDs, the voltage distribution approximately scales according to the properties (Eqs. 25 and 29 ), but does not scale correctly for small $\sigma_{v}$. Accordingly, we expect that the LN models will show nearly perfect gain scaling for larger input strengths, but that scaling will break down for small $\sigma_{\mathrm{v}}$. This is confirmed in Figure $8 A$. Note that Equation 25 holds for a range of voltages that are part of the spike event: gain scaling is insensitive to the exact definition of $v_{\text {spike. }}$.

that the later expression of certain subthreshold potassium conductances (e.g., $I_{\mathrm{A}}$ ) may reduce the inherent gain-scaling property of the spike-generating mechanism. As intrinsic properties shift with expression of channels with specific functional roles (Connors and Gutnick, 1990; Maravall et al., 2007; Díaz-Quesada and Maravall, 2008), gain-scaling behavior might be supported by a different mechanism, such as the slow AHP current observed in the adult barrel cortex (Díaz-Quesada and Maravall, 2008).
In the conductance-based model, higher $G_{\mathrm{Na}} / G_{\mathrm{K}}$ led to better gain scaling, but $G_{\mathrm{Na}} / G_{\mathrm{K}}>\sim 2$ led to spontaneous firing in which a spike carries little information about the input stimulus. Gainscaling mature neurons typically had a $G_{\mathrm{Na}} / G_{\mathrm{K}}$ ratio of close to 1 and were not spontaneously active. We would speculate that in vitro, the particular current ratio of $I_{\mathrm{Na}} / I_{\mathrm{K}}=1$ may promote intrinsic gain-scaling ability while maintaining feature selectivity and sparse, informative firing. 
Previously, contributions to gain scaling in single neurons have been identified as arising from different biophysical properties. In retinal ganglion cells, gain control has been attributed to slow sodium inactivation leading to a $\sigma$-dependent build-up of inactivation (Kim and Rieke, 2001). In adult barrel cortex neurons, contributions to gain scaling have been associated with a calcium-activated potassium conductance underlying slow afterspike hyperpolarization (Díaz-Quesada and Maravall, 2008). In these cases, the effect was attributed to a relatively slow-timescale activity-dependent current that reduced threshold in the presence of increased neuronal activity. Such slow processes might serve to shift the mean of the subthreshold distribution.

However, gain scaling can be achieved without slow processes, for example, if the underlying system is described by an LN model that exhibits a power-law nonlinearity (Miller and Troyer, 2002). In this case Gaussian stimuli with different variances expose different ranges of the power-law input/output function that, when scaled by $\sigma$, collapse to a common curve. This is not the case in our data; the input/output relations are non-power-law for both the data and the EIF model that matches the data. In our study, we observe gain-scaling behavior with only fast spike-generating currents and no slow processes (Fig. 7). The gain scaling that we observed does not rely on the specific form of the nonlinearity, but emerges from the approximate subthreshold linearity of the leak current and the way in which the fast spike-generating currents shape how the input probes the dynamics in voltage state space. The mechanism that we have identified here holds for models that are one dimensional in the sense that a single dynamical variable (the voltage) is sufficient to account for the dynamics. Generally such models correspond to LN models with a single filter (Hong et al., 2007). The same conditions could in principle hold in a multidimensional dynamical model, but it would depend on the properties and geometry of the dynamical threshold in that multidimensional space (Hong et al., 2007) and is likely to be less generic.

While effects of stimulus mean and variance on LN model components have been noted before in single neurons without slow timescales (Agüera y Arcas et al., 2003; Yu and Lee, 2003; Paninski et al., 2004; Gaudry and Reinagel, 2007), to our knowledge, this paper is the first report of perfect gain scaling measured experimentally in single neurons. This manifestation of gain scaling depends on the kinetics of the spike-generating currents.

The linear dependence of firing rate on input SD alongside gain scaling implies that the feature selection computation in simple neurons is an example of a multiplexed temporal code (Fairhall et al., 2001; Wark et al., 2007; Lundstrom et al., 2008; Panzeri et al., 2010): the mean rate averaged over many spikes carries information about stimulus context, $\sigma$, while individual spikes carry information about fluctuations in the filtered stimulus. This multiplexed code can be generated with a single active mechanism because the membrane time constant determined by the leak is long compared with the duration for which the spikegenerating currents are active. The mean state of the neuron between spikes is only sensitive to the mean rate, and so there is still freedom for precise modulation of the spike times on shorter time scales.

Previous studies have explored the robustness of the outputs of neurons and neural networks with respect to variations in the high-dimensional space of single neuron parameters (Marder and Goaillard, 2006). Here, we quantified a neuron's output properties not in terms of specific firing patterns but by its ability to exhibit gain scaling, and found that this property is supported by a one-dimensional set of conductance parameters- the ratio of maximal sodium to potassium currents. This suggests that a partial tuning of intrinsic parameters occurs during development. This tuning may contribute to the shift in information processing that occurs as the cortex prepares for the onset of sensory experience.

While the input-output properties of a single neuron are a function of synapses, morphology, and ion channel properties, here we tested only the developing gain-scaling properties of the somatic spike-generating mechanism. The developmental period we investigated is also characterized by significant synaptic maturation (Garaschuk et al., 2000; McCabe et al., 2007; Feldmeyer and Radnikow, 2009); determining how changing synaptic inputs interact with the developing gain-scaling properties of single cortical neurons is an open question. It is possible that intrinsic somatic properties can serve to alleviate or normalize out the effects of changes in synaptic filtering that help to shape the statistics of the input to the soma.

Numerous studies have shown that developing neural systems display activity with no apparent sensory correlate-so-called "spontaneous activity"-and this activity can trigger developmental changes ranging from ion channel expression to network connectivity (Moody and Bosma, 2005). In the neonatal mouse, waves of spontaneous activity are initiated in a discrete pacemaker region in the ventrolateral cortex (Lischalk et al., 2009) and propagate across the cortex from E17-P2 (Corlew et al., 2004; McCabe et al., 2006). Our results show that neurons at these early stages do not adjust excitability to compensate for the variance of input amplitudes. It is possible that this lower propensity for spontaneous firing, combined with higher intrinsic excitability in response to large inputs, may contribute to the ability of immature neurons to respond to pacemaker input by participating in spontaneous waves of activity, while at the same time avoiding asynchronous firing between waves of activity (Lischalk et al., 2009; Conhaim et al., 2010, 2011). In contrast, mature neurons responded to a wider range of stimuli (Fig. $3 B$ ) and so may be less selective for widespread network activity. Thus, developmental changes in gain scaling may be part of a process that changes cortical neurons from participants in waves of spontaneous activity that are essential for cortical development into asynchronous and efficient information-processing computational units.

Computational characterizations of single neurons have demonstrated that sophisticated coding properties can arise from the combined diversity of morphology and ion channel properties. We found that in the developing cortex, single neurons exhibit gain scaling well before the cortical network reaches an adult state. Furthermore, the interaction of basic voltage-gated channels that gives rise to spikes provides an intrinsic mechanism for adaptive coding. Within a developmental context, the emergence of gain scaling may serve to reduce the early propensity of cortical neurons to entrain in large-scale spontaneous patterns of activity and thus terminate such activity on the appropriate developmental schedule. These results underscore the rich intrinsic computational repertoire of single neurons.

\section{References}

Abbott L, Kepler T (1990) Model neurons: from Hodgkin-Huxley to Hopfield. In: Statistical mechanics of neural networks, pp 5-18. New York: Springer.

Agüera y Arcas B, Fairhall AL, Bialek W (2003) Computation in a single neuron: Hodgkin and Huxley revisited. Neural Comput 15:1715-1749. CrossRef Medline

Atick JJ (2011) Could information theory provide an ecological theory of sensory processing. Network 22:4-44. CrossRef Medline 
Attneave F (1954) Some informational aspects of visual perception. Psychol Rev 61:183-193. CrossRef Medline

Badel L, Lefort S, Berger TK, Petersen CC, Gerstner W, Richardson MJ (2008a) Extracting nonlinear integrate-and-fire models from experimental data using dynamic I-V curves. Biol Cybern 99:361-370. CrossRef Medline

Badel L, Lefort S, Brette R, Petersen CC, Gerstner W, Richardson MJ (2008b) Dynamic I-V curves are reliable predictors of naturalistic pyramidalneuron voltage traces. J Neurophysiol 99:656-666. CrossRef Medline

Barlow H (1961) Possible principles underlying the transformation of sensory messages. In: Sensory communication (Rosenblith W, ed), pp 217234. Cambridge, MA: MIT.

Bean BP (2007) The action potential in mammalian central neurons. Nat Rev Neurosci 8:451-465. CrossRef Medline

Brenner N, Strong SP, Koberle R, Bialek W, de Ruyter van Steveninck RR (2000) Synergy in a neural code. Neural Comput 12:1531-1552. CrossRef Medline

Brette R, Piwkowska Z, Rudolph M, Bal T, Destexhe A (2007) A nonparametric electrode model for intracellular recording. Neurocomputing 70 : 1597-1601. CrossRef

Bryant HL, Segundo JP (1976) Spike initiation by transmembrane current: a white-noise analysis. J Physiol 260:279-314. Medline

Carandini M, Heeger DJ (2012) Normalization as a canonical neural computation. Nat Rev Neurosci 13:51-62. Medline

Conhaim J, Cedarbaum ER, Barahimi M, Moore JG, Becker MI, Gleiss H, Kohl C, Moody WJ (2010) Bimodal septal and cortical triggering and complex propagation patterns of spontaneous waves of activity in the developing mouse cerebral cortex. Dev Neurobiol 70:679-692. CrossRef Medline

Conhaim J, Easton CR, Becker MI, Barahimi M, Cedarbaum ER, Moore JG, Mather LF, Dabagh S, Minter DJ, Moen SP, Moody WJ (2011) Developmental changes in propagation patterns and transmitter dependence of waves of spontaneous activity in the mouse cerebral cortex. J Physiol 589:2529-2541. CrossRef Medline

Connors BW, Gutnick MJ (1990) Intrinsic firing patterns of diverse neocortical neurons. Trends Neurosci 13:99-104. CrossRef Medline

Corlew R, Bosma MM, Moody WJ (2004) Spontaneous, synchronous electrical activity in neonatal mouse cortical neurones. J Physiol 560:377-390. CrossRef Medline

de Boer E, Kuyper P (1968) Triggered correlation. Biomed Eng IEEE Trans 15:169-179.

Díaz-Quesada M, Maravall M (2008) Intrinsic mechanisms for adaptive gain rescaling in barrel cortex. J Neurosci 28:696-710. CrossRef Medline

Ermentrout B (1998) Linearization of f-I curves by adaptation. Neural Comput 10:1721-1729. CrossRef Medline

Fairhall AL, Lewen GD, Bialek W, de Ruyter Van Steveninck RR (2001) Efficiency and ambiguity in an adaptive neural code. Nature 412:787-792. CrossRef Medline

Famulare M, Fairhall A (2010) Feature selection in simple neurons: how coding depends on spiking dynamics. Neural Comput 22:581-598. CrossRef Medline

Famulare MG, Fairhall AL (2011) Adaptive probabilistic neural coding from deterministic spiking neurons: analysis from first principles. Eprint arxiv.org/abs/1111.0097.

Feldmeyer D, Radnikow G (2009) Developmental alterations in the functional properties of excitatory neocortical synapses. J Physiol 587:18891896. CrossRef Medline

Fourcaud N, Brunel N (2002) Dynamics of the firing probability of noisy integrate-and-fire neurons. Neural Comput 14:2057-2110. CrossRef Medline

Fourcaud-Trocmé N, Hansel D, van Vreeswijk C, Brunel N (2003) How spike generation mechanisms determine the neuronal response to fluctuating inputs. J Neurosci 23:11628-11640. Medline

Garaschuk O, Linn J, Eilers J, Konnerth A (2000) Large-scale oscillatory calcium waves in the immature cortex. Nat Neurosci 3:452-459. CrossRef Medline

Gaudry KS, Reinagel P (2007) Contrast adaptation in a nonadapting LGN model. J Neurophysiol 98:1287-1296. CrossRef Medline

Gerstner W, Kistler WM (2002) Spiking neuron models: single neurons, populations, plasticity. Cambridge, UK: Cambridge UP.

Hines ML, Carnevale NT (1997) The NEURON simulation environment. Neural Comput 9:1179-1209. CrossRef Medline
Hodgkin A, Huxley A (1952) A quantitative description of membrane current and its application to conduction and excitation in nerve. J Neurophysiol 117:500-544.

Hong S, Agüera y Arcas B, Fairhall AL (2007) Single neuron computation: from dynamical system to feature detector. Neural Comput 19:31333172. CrossRef Medline

Hunter IW, Korenberg MJ (1986) The identification of nonlinear biological systems: Wiener and Hammerstein cascade models. Biol Cybern 55:135144. Medline

Izhikevich E (2007) Dynamical systems in neuroscience: the geometry of excitability and bursting. Cambridge, MA: MIT.

Jolivet R, Lewis TJ, Gerstner W (2004) Generalized integrate-and-fire models of neuronal activity approximate spike trains of a detailed model to a high degree of accuracy. J Neurophysiol 92:959-976. CrossRef Medline

Kepler T, Abbott L, Marder E (1992) Reduction of conductance-based neuron models. Biol Cybern 381-387.

Kim KJ, Rieke F (2001) Temporal contrast adaptation in the input and output signals of the salamander retinal ganglion cells. J Neurosci 21:287299. Medline

Kistler WM, Gerstner W, van Hemmen JL (1997) Reduction of the Hodgkin-Huxley equations to a single-variable threshold model. Neural Comput 9:1015-1045. CrossRef

Laughlin S (1981) A simple coding procedure enhances a neuron's information capacity. Z Naturforsch C 36:910-912. Medline

Lin J (1991) Divergence measures based on the Shannon entropy. IEEE Trans Inform Theory 37:145-151. CrossRef

Lischalk JW, Easton CR, Moody WJ (2009) Bilaterally propagating waves of spontaneous activity arising from discrete pacemakers in the neonatal mouse cerebral cortex. Dev Neurobiol 69:407-414. CrossRef Medline

Lundstrom BN, Higgs MH, Spain WJ, Fairhall AL (2008) Fractional differentiation by neocortical pyramidal neurons. Nat Neurosci 11:1335-1342. CrossRef Medline

Lundstrom BN, Fairhall AL, Maravall M (2010) Multiple timescale encoding of slowly varying whisker stimulus envelope in cortical and thalamic neurons in vivo. J Neurosci 30:5071-5077. CrossRef Medline

Mainen ZF, Joerges J, Huguenard JR, Sejnowski TJ (1995) A model of spike initiation in neocortical pyramidal neurons. Neuron 15:1427-1439. CrossRef Medline

Maravall M, Petersen RS, Fairhall AL, Arabzadeh E, Diamond ME (2007) Shifts in coding properties and maintenance of information transmission during adaptation in barrel cortex. PLoS Biol 5:e19. CrossRef Medline

Marder E, Goaillard JM (2006) Variability, compensation and homeostasis in neuron and network function. Nat Rev Neurosci 7:563-574. CrossRef Medline

Massengill JL, Smith MA, Son DI, O’Dowd DK (1997) Differential expression of K4-AP currents and Kv3.1 potassium channel transcripts in cortical neurons that develop distinct firing phenotypes. J Neurosci 17:31363147. Medline

McCabe AK, Chisholm SL, Picken-Bahrey HL, Moody WJ (2006) The selfregulating nature of spontaneous synchronized activity in developing mouse cortical neurones. J Physiol 577:155-167. CrossRef Medline

McCabe AK, Easton CR, Lischalk JW, Moody WJ (2007) Roles of glutamate and GABA receptors in setting the developmental timing of spontaneous synchronized activity in the developing mouse cortex. Dev Neurobiol 67:1574-1588. CrossRef Medline

McCormick DA, Prince DA (1987) Post-natal development of electrophysiological properties of rat cerebral cortical pyramidal neurones. J Physiol 393:743-762. Medline

Miller KD, Troyer TW (2002) Neural noise can explain expansive, powerlaw nonlinearities in neural response functions. J Neurophysiol 87:653659. Medline

Moody WJ, Bosma MM (2005) Ion channel development, spontaneous activity, and activity-dependent development in nerve and muscle cells. Physiol Rev 85:883-941. CrossRef Medline

Paninski L, Lau B, Reyes A (2003) Noise-driven adaptation: in vitro and mathematical analysis. Neurocomputing 52:877-883.

Paninski L, Pillow JW, Simoncelli EP (2004) Maximum likelihood estimation of a stochastic integrate-and-fire neural encoding model. Neural Comput 16:2533-2561. CrossRef Medline

Panzeri S, Brunel N, Logothetis NK, Kayser C (2010) Sensory neural codes using multiplexed temporal scales. Trends Neurosci 33:111-120. CrossRef Medline 
Picken-Bahrey HL, Moody WJ (2003a) Early development of voltage-gated ion currents and firing properties in neurons of the mouse cerebral cortex. J Neurophysiol 89:1761-1773. CrossRef Medline

Picken-Bahrey HL, Moody WJ (2003b) Voltage-gated currents, dye and electrical coupling in the embryonic mouse neocortex. Cereb Cortex 13: 239-251. Medline

Pillow J, Simoncelli E (2003) Biases in white noise analysis due to nonpoisson spike generation. Neurocomputing 52:109-115.

Powers RK, Dai Y, Bell BM, Percival DB, Binder MD (2005) Contributions of the input signal and prior activation history to the discharge behaviour of rat motoneurones. J Physiol 562:707-724. Medline

Rieke F, Warland D, de Ruyter van Steveninck R, Bialek W (1996) Spikes: exploring the neural code. Cambridge, MA: MIT.

Ringach DL, Malone BJ (2007) The operating point of the cortex: neurons as large deviation detectors. J Neurosci 27:7673-7683. CrossRef Medline

Risken H (1996) The Fokker-Planck equation: materials and methods of solution and applications, Ed 2. Berlin: Springer.

Sanes DH, Reh TA, Harris WA (2006) Development of the nervous system. Burlington, MA: Elsevier Academic.

Sharpee T, Rust NC, Bialek W (2004) Analyzing neural responses to natural signals: maximally informative dimensions. Neural Comput 16:223-250. CrossRef Medline
Slee SJ, Higgs MH, Fairhall AL, Spain WJ (2005) Two-dimensional time coding in the auditory brainstem. J Neurosci 25:9978-9988. CrossRef Medline

Svirskis G, Dodla R, Rinzel J (2003) Subthreshold outward currents enhance temporal integration in auditory neurons. Biol Cybern 89:333-340. CrossRef Medline

Theunissen FE, Sen K, Doupe AJ (2000) Spectral-temporal receptive fields of nonlinear auditory neurons obtained using natural sounds. J Neurosci 20:2315-2331. Medline

Touboul J (2009) Importance of the cutoff value in the quadratic adaptive integrate-and-fire model. Neural Comput 21:2114-2122. CrossRef Medline

Vacher H, Mohapatra DP, Trimmer JS (2008) Localization and targeting of voltage-dependent ion channels in mammalian central neurons. Physiol Rev 88:1407-1447. CrossRef Medline

Wark B, Lundstrom BN, Fairhall A (2007) Sensory adaptation. Curr Opin Neurobiol 17:423-429. CrossRef Medline

Yu Y, Lee TS (2003) Dynamical mechanisms underlying contrast gain control in single neurons. Phys Rev E Stat Nonlin Soft Matter Phys 68:011901. CrossRef Medline 